\title{
Microstructurally-sensitive fatigue crack nucleation in Ni-based single and oligo crystals
}

\author{
Bo Chen ${ }^{1}$, Jun Jiang1,2, Fionn P.E. Dunne ${ }^{1,2}$ \\ ${ }^{1}$ Department of Materials, ${ }^{2}$ Department of Mechanical Engineering, Imperial \\ College London, SW7 2AZ, UK
}

\begin{abstract}
An integrated experimental, characterization and computational crystal plasticity study of cyclic plastic beam loading has been carried out for nickel single crystal (CMSX4) and oligocrystal (MAR002) alloys in order to assess quantitatively the mechanistic drivers for fatigue crack nucleation.
\end{abstract}

The experimentally validated modelling provides knowledge of key microstructural quantities (accumulated slip, stress and GND density) at experimentally observed fatigue crack nucleation sites and it is shown that while each of these quantities is potentially important in crack nucleation, none of them in its own right is sufficient to be predictive. However, the local (elastic) stored energy density, measured over a length scale determined by the density of SSDs and GNDs, has been shown to predict crack nucleation sites in the single and oligocrystals tests. In addition, once primary nucleated cracks develop and are represented in the crystal model using XFEM, the stored energy correctly identifies where secondary fatigue cracks are observed to nucleate in experiments. This (Griffith-Stroh type) quantity also correctly differentiates and explains intergranular and transgranular fatigue crack nucleation.

\section{Research highlights}

- Accumulated slip, GND density and bending stress are essential but not sufficient in their own right to drive fatigue crack nucleation.

- Local stored energy density identifies all fatigue crack nucleation sites in the single and oligocrystals.

- Stored energy differentiates between transgranular and intergranular crack nucleation observed in oligocrystal specimens.

- Secondary fatigue crack nucleation in single crystals has been explained by stored energy criterion.

Keywords: fatigue, crack nucleation, nickel, crystal plasticity, EBSD 


\section{Introduction}

High-performance nickel-based superalloys are increasingly used as safety critical rotatory components in the vehicle, power and aerospace industries because of their outstanding mechanical properties. In service, these components often undergo cyclic loading at high temperature and thus fatigue fractures are a dominating failure mode [1]. Generally, fatigue failures can be divided into crack nucleation and propagation stages. Fatigue crack propagation can be reasonably accurately predicted $[2,3]$. However conservative fatigue life prediction methods are currently often used in component design methodologies due to the large scatter of the observed fatigue life measurements argued to originate from the microstructural sensitivity of crack nucleation [4]. Due to the complexity of microstructure and the difficulty of direct measurement of the deformation quantities at such small scales, there remains a clear need to establish mechanistic understanding and hence quantitative predictive capability for crack nucleation processes $[5,6]$. The aim of this paper is to enhance the understanding of fatigue crack nucleation processes in nickel alloys by combining state of the art characterization techniques (e.g. high resolution digital image correlation) with quantitatively validated crystal plasticity finite element (CPFE) microstructure modelling. A related objective is to establish predictive capabilities for both the nucleation site of fatigue crack nucleation as well as the number of cycles in order to cause crack nucleation in the nickel-based superalloys considered.

Fatigue cracking in FCC nickel-based superalloys has been studied by many authors e.g. $[7,8]$. In these studies, micro fatigue cracks at persistent slip bands (PSBs) were observed. The dislocations within the developing PSBs roughened the free surfaces and led to fatigue crack nucleation. Ultimately, local accumulated slip contributes to the formation of cracks. In addition, the total energy related to PSB formation has been associated with fatigue crack nucleation (FCN) in nickelbased alloys, which has been studied in $[9,10]$. These authors calculated the energy of PSBs and defined a failure criterion based on the stability of dislocation movement. The associated critical PSB energy has been used to determine the sites of FCN. Their work has also shown that fatigue cracks tend to nucleate at slip localization sites where the dissipated energy is high. Hence, it is argued that the PSBs and localized slip accumulation (plastic strain) are necessary (but not sufficient) factors which lead to fatigue crack nucleation [7, 11]. Moreover, grains with low misorientation angle and high Schmid factors were found to be the potential locations of fatigue crack nucleation by other authors [12]. It has also been pointed out that the elastic and plastic anisotropies determine the stress and strain distributions at local scale and thus influence strongly the fatigue crack nucleation [13, 14]. Fatigue crack nucleation has also been associated with microstructural features such as grain boundaries $[15,16]$ including special grain boundaries e.g. twin boundaries $[17,18]$. Miao et al showed that higher energy grain boundaries tend to have larger lattice misorientations and dislocations are more likely to pile up at high-angle grain boundaries. However, in the case of nickel-based superalloys, cracks often nucleate along preferred-orientated $\sum 3$ twin boundaries embedded in large grains. The distributions of grain size in dual microstructural disk alloys has been investigated in $[19,20]$. This work shows that the samples from coarse grain areas of the bulk show shorter fatigue life compared with the samples from finer grain areas and large grains tend to nucleate crystallographic grain facets where failure is most likely to originate. More 
recently, FCN in nickel-based RS5 has been studied by Wan et al [21]. They utilised a fatigue crack nucleation criterion based on local stored energy [4] which considers the combined effects of local accumulated slip, flow stress and dislocation density.

The FCN mechanisms occurring in other alloy systems are often similar. For example, in FCC TiAl alloys the interactions between dislocations and grain boundaries or twin boundaries have been associated with fatigue crack nucleation [22]. In [23], the activated slip systems in Ti alloy under fatigue tests are found mostly to be of basal and prismatic slip orientation, and micro-textured areas are often found to be the initiation sites of fatigue cracks. The relationships between slip banding, Schmid factor and crack nucleation in 316L stainless steel have been investigated in [24]. These results show that most observed cracks nucleated within slip bands or at grain boundaries. About two thirds of these cracks nucleate in slip systems with the highest Schmid factor, which have an orientation of approximately $45^{\circ}$ in relation to the loading axis. Much related research demonstrates that grain size and crystallographic orientation have a significant influence on crack formation [25].

It is therefore argued that fatigue crack nucleation is associated with key factors including accumulated slip, the formation of PSBs; GNDs that result from plastic strain gradients, localized stress and local stored energy. In addition, microstructural features such as agglomerates/inclusions, annealing twins, grain size distribution, inherent defects, and grain boundaries play a significant role in fatigue behaviour, because they significantly influence these local microstructural quantities. Some progress has therefore been made in understanding the mechanistic basis of fatigue crack nucleation and its relationship to local microstructural features. However, a quantitatively predictive model is likely still some way off.

In a previous paper [26], combined high-resolution digital image correlation with crystal plasticity (CP) modelling of single and oligocrystal three-point cyclic bend tests enabled a highly detailed investigation of slip activation, its spatial reach, the resulting strain distributions and slip localisation to be measured. These results were compared with the CP model predictions, providing persuasive evidence that quantitative predictive capability exists. However, this work did not address subsequent cycling leading to fatigue crack nucleation and growth in the Ni single and oligocrystal samples. Hence the new aspects in this paper are experimental fatigue tests through to crack nucleation and growth, the introduction and description of a stored energy criterion for crack nucleation, its implementation in to the CP material model and prediction of crack nucleation sites in single (CMSX4) and oligocrystal (MAR002) Ni bend fatigue samples, and comparison with experimental observations. In addition, at the sites of observed cracks in the Ni single crystals, XFEM has been utilized to introduce nucleated cracks in to the $\mathrm{CP}$ modelling in order to assess the resulting redistribution of stored energy to demonstrate that the experimentally observed secondary crack nucleation in the $\mathrm{Ni}$ single crystal samples also results at the consequent highest locations of stored energy.

In this paper, we therefore present a systematic fatigue study of crystallographic and grain-to-grain interaction effects by examining single crystal CMSX04 and oligocrystal MAR002 samples under three-point bending low cycle fatigue. 
Electron backscatter diffraction (EBSD) and HR-DIC were used to characterize the crystal orientations, developing distributions of geometrically necessary dislocations (GNDs), and the slip localisations with respect to crack nucleation sites. Microstructurally representative crystal plasticity finite element models were developed to capture local stress, strain, and dislocation density evolutions occurring over a number of fatigue loading cycles. These models, directly validated by HR-DIC measurements, are utilised to investigate the stored energy criterion for fatigue crack nucleation in the single and oligocrystal test samples. XFEM analyses also enable the investigation of nucleated cracks and their role in the redistribution of stored energy in order to assess observed secondary cracking. The CP and XFEM predicted and experimentally observed fatigue crack nucleation sites are compared and assessed.

\section{Methodology}

\subsection{Sample preparation and three-point bending test}

Two CMSX4 single crystal and three MAR002 oligo-crystal nickel-based superalloy bars were provided by Rolls-Royce to study fatigue crack nucleation behaviour. These samples were cut into rectangular shape with dimensions of $\sim 12 \times 3 \times 3 \mathrm{~mm}^{3}$ using electro discharge machining. Dimension details are given in Fig 1 along with a schematic of the three-point beam loading and the loading rig. The front and back surfaces of these specimens were polished using a series of grit silicon carbide papers (800, 1200, 2000 and 4000 respectively). For EBSD characterization, the front surfaces were finished with colloidal silica suspension for 30 minutes to remove the surface deformation and residual stress. A layer of nano-size colloidal silica $(250 \mathrm{~nm})$, was coated over the back surface region of interest (ROI) for subsequent HR-DIC measurement.

(a)

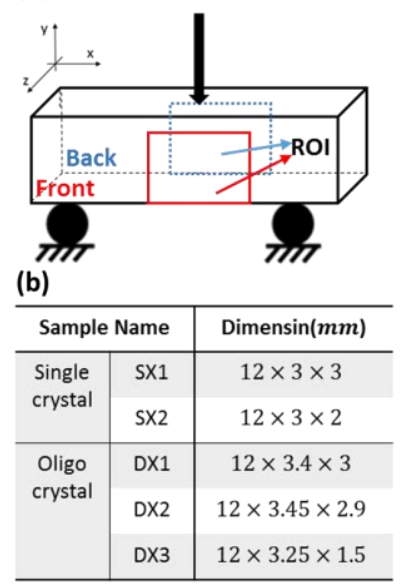

(c)

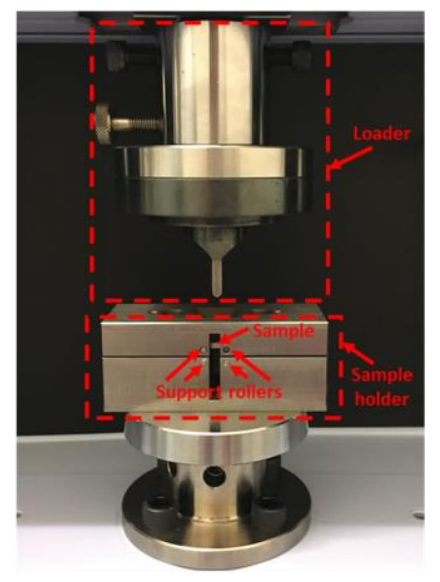

(d)

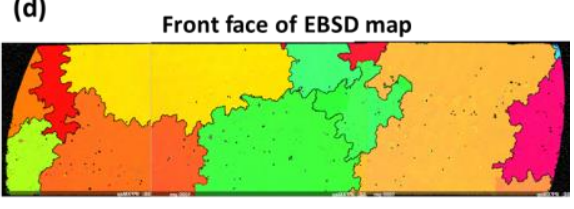

Back face of EBSD map

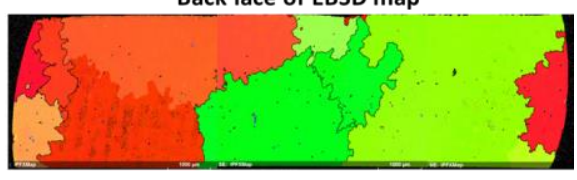

Figure 1. (a) Schematic diagram of specimen geometry with marked regions of interest (ROI). The detailed dimensions are shown in (b); the three-point bending mechanical testing rig is shown in (c). An example oligocrystal is shown in (d).

The crystal orientation and strain at the ROIs shown in Fig. 1 were characterized and studied using EBSD and HR-DIC as discussed in [26], and the ROIs are marked using red and blue respectively. The three-point bending tests were carried out on a SHIMADZU mechanical testing machine. The cyclic loading was applied with a loading ratio of $\mathrm{R}=0.1$, and the loading history for each sample has been detailed 
in Table 1. Both single and oligocrystal samples have been investigated where for clarity, an oligocrystal (shown in Figure 1(d)) is in this paper defined to be a polycrystalline sample containing a small number of grains which are prismatic through the sample thickness with respect to both morphology and crystallography. For single crystal SX1, and for directionally solidified oligocrystals DX1 and DX2, at the end of the prior loading specified, the maximum loads were increased to a higher peak magnitude for all-subsequent cyclic loading to generate fatigue cracks in the regime of low cycle fatigue. Samples SX2 and DX3 were cyclically loaded throughout at the same peak load. Fig 1(c) shows the experimental three-point bending set-up. The test was interrupted after a given number of cycles, so samples were removed from the testing rig at selected intervals and inserted into an SEM chamber to capture SEM images for HR-DIC analysis.

Table 1. The sample loading histories

\begin{tabular}{|c|c|c|c|}
\hline \multicolumn{2}{|c|}{$\begin{array}{l}\text { CMSX4 and MAROO2 } \\
\text { Sample No. }\end{array}$} & \multicolumn{2}{|c|}{$\begin{array}{l}\text { Maximum/minimum cyclic load }(\mathrm{N}) \\
\text { (Total No. Fatigue cycles) }\end{array}$} \\
\hline & & Prior & Subsequent loading \\
\hline \multirow{2}{*}{$\begin{array}{l}\text { Single } \\
\text { crystals }\end{array}$} & SX1 & $\begin{array}{c}3000 / 300 \\
\text { (800 Cycles) }\end{array}$ & $\begin{array}{c}3700 / 370 \\
(12000 \text { Cycles) }\end{array}$ \\
\hline & SX2 & \multicolumn{2}{|c|}{$\begin{array}{c}2500 / 250 \\
(36450 \text { Cycles) }\end{array}$} \\
\hline \multirow{3}{*}{$\begin{array}{l}\text { Oligo } \\
\text { crystals }\end{array}$} & DX1 & $\begin{array}{c}3000 / 300 \\
\text { (800 Cycles) }\end{array}$ & $\begin{array}{c}4000 / 400 \\
\text { (5550 Cycles) }\end{array}$ \\
\hline & $\mathrm{DX} 2$ & $\begin{array}{l}2700 / 270 \\
\text { (620 Cycles) }\end{array}$ & $\begin{array}{c}4050 / 405 \\
\text { (5000 Cycles) }\end{array}$ \\
\hline & DX3 & \multicolumn{2}{|c|}{$\begin{array}{c}3500 / 350 \\
(4500 \text { Cycles) }\end{array}$} \\
\hline
\end{tabular}

Explicit microstructurally faithful CPFE models for the single and oligocrystal beams were also developed to mimic the experimental loading histories, thus enabling direct and unambiguous comparison between the modelling and the experimental results.

\subsection{HR-DIC measurement}

The HR-DIC technique enables the determination of full field, in-plane strain by calculating the relative shifts of speckle patterns within a region of interest (ROI) between two successive testing images. As there is no size limit for the images if the contrast and resolution are high enough to ensure the accuracy, this technique is used in determining total strain at the local microstructural scale. The accuracy of the DIC strain measurement is estimated as $\sim 0.1 \%$ [27]. The detailed development and principles of this technique can be found elsewhere [27-30].

Reference SEM images of the speckled ROI were collected in the undeformed state using an accelerating voltage of $20 \mathrm{kV}$ with a working distance of around $15 \mathrm{~mm}$. The brightness and contrast were carefully adjusted to ensure the white silica particles could be clearly seen as shown in Fig. 2. DIC analysis was conducted using in-house developed Matlab scripts in which the rigid body rotation and translation between frames were removed to improve the accuracy of strain measurement [29-31]. $100 \times 100$ pixels of the subset windows with an overlap of $90 \%$ were used as local strain gauges to measure and calculate strain maps [26]. 


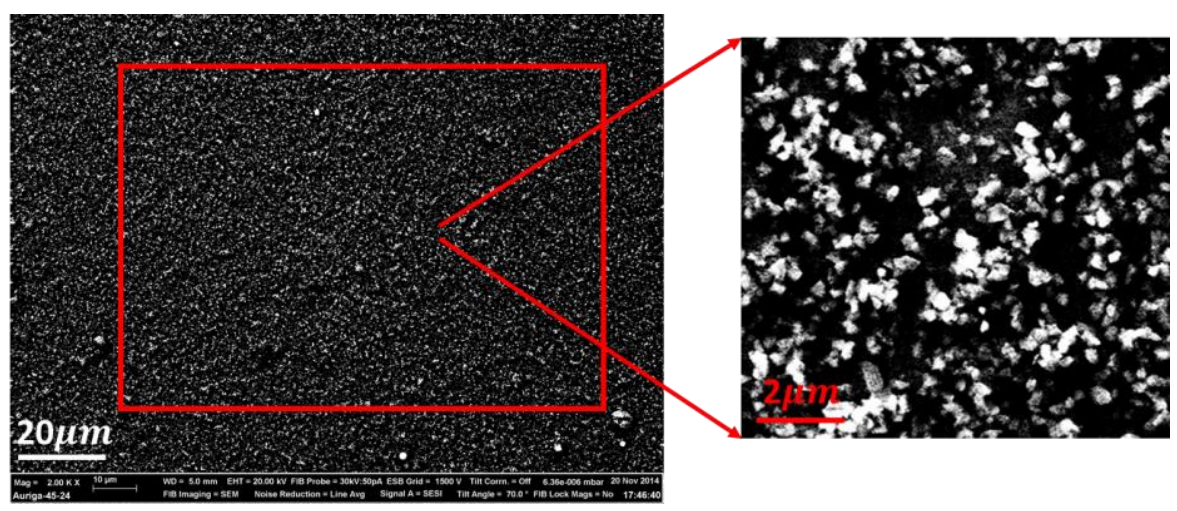

Figure 2. A layer of silica suspension is coated on the sample free surface (single crystal SX1) to perform DIC measurement.

\subsection{Crystal plasticity finite element modelling}

\subsubsection{Crystal plasticity modelling}

In the previous paper [26], crystal plasticity modelling has been developed for single and oligocrystal nickel alloys identical to those considered in this work. The calibration of the crystal model for the alloys, and the full-field, grain-level validation was also presented for small numbers of loading cycles, in the absence of fatigue crack nucleation. The model was demonstrated to capture single and multiple slip system activation, the spatial distributions of the slip lines on the free surfaces, the slip accumulation due to cyclic loading, and the full-field heterogeneous strain distributions. Hence a brief summary only is included here. The crystal plasticity equations are rate-dependent and implemented in the user material subroutine UMAT using ABAQUS standard/explicit analysis $[7,21,26,27$, 32]. The total deformation gradient $\mathbf{F}$ is split into elastic $\mathbf{F}^{\mathbf{e}}$ and plastic $\mathbf{F}^{\mathbf{p}}$ deformation gradient terms, where $\mathbf{F}^{\mathbf{e}}$ is responsible for lattice rotation and stretch while $\mathbf{F}^{\mathbf{p}}$ contributes to slip and continuum rotation.

$$
\mathbf{F}=\mathbf{F}^{\mathbf{e}} \mathbf{F}^{\mathbf{p}}
$$

The plastic velocity gradient $\mathbf{L}^{\mathbf{p}}$ is given by

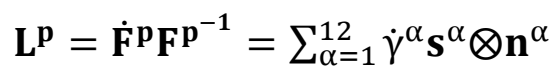

where $\mathbf{s}^{\alpha}$ and $\mathbf{n}^{\alpha}$ are the line vectors along slip direction and slip plane normal of slip system $\alpha$ respectively, and the slip rate $\dot{\gamma}^{\alpha}$ on a given slip system $\alpha$ is expressed by

$$
\dot{\gamma}^{\alpha}=\rho_{\mathrm{ssdm}} v b^{2} \exp \left(-\frac{\Delta \mathrm{F}}{\mathrm{kT}}\right) \sinh \left(\frac{\Delta V}{\mathrm{kT}}\left|\tau^{\alpha}-\tau_{\mathrm{c}}{ }^{\alpha}\right|\right)
$$

in which $\rho_{\text {ssdm }}$ is the mobile dislocation density, $b$ the magnitude of Burger's vector, $v$ the jump frequency for driving thermally activated dislocation escape, $\Delta \mathrm{F}$ the activation energy, $\Delta \mathrm{V}$ the associated activation volume, $\tau^{\alpha}$ the resolved shear stress and $\tau_{\mathrm{c}}{ }^{\alpha}$ is the corresponding slip system critical resolved shear stress.

In this study, a modified Taylor hardening rule is used to account for isotropic hardening

$$
\tau_{\mathrm{c}}{ }^{\alpha}=\tau_{\mathrm{c} 0}{ }^{\alpha}+\mathrm{G}_{12} \cdot \mathrm{b} \cdot \sqrt{\rho_{\mathrm{GND}}+\rho_{\mathrm{SSD}}}
$$

in which $\tau_{\mathrm{c} 0}{ }^{\alpha}$ is the initial slip strength, $\mathrm{G}_{12}$ the shear modulus, and $\rho_{\mathrm{SSD}}$ and $\rho_{\mathrm{GND}}$ are the statistically stored and geometrically necessary dislocation densities 
respectively. The evolution of SSDs is simply taken to evolve linearly with accumulated slip rate as follows

$$
\dot{\rho}_{\mathrm{SSD}}=\lambda \cdot \dot{\mathrm{p}}
$$

where $\lambda$ is the hardening coefficient, and the effective plastic strain rate $\dot{\mathrm{p}}$ is given by

$$
\dot{\mathrm{p}}=\left(\frac{2}{3} \varepsilon^{\mathrm{p}}: \varepsilon^{\mathrm{p}}\right)^{1 / 2}
$$

The evolution of SSDs and GNDs [33] gives rise to the development of shear resistance on each slip system., for which the GNDs are generated by strain gradients, which accommodate the lattice curvature in the material. Nye's dislocation tensor $\boldsymbol{\Lambda}$ is used to compute the components of GNDs, given by

$$
\boldsymbol{\Lambda}=\operatorname{curl}\left(\mathbf{F}^{\mathbf{P}}\right)=\sum_{\alpha=1}^{12} \rho_{\mathrm{Gs}}^{\alpha} \mathbf{b}^{\alpha} \otimes \mathbf{s}^{\alpha}+\rho_{\mathrm{Get}}^{\alpha} \mathbf{b}^{\alpha} \otimes \mathbf{t}^{\alpha}+\rho_{\mathrm{Gen}}^{\alpha} \mathbf{b}^{\alpha} \otimes \mathbf{n}^{\alpha}
$$

in which $\rho_{\mathrm{Gs}}^{\alpha}$ are screw dislocation components on slip system $\alpha$ with line vector along the slip direction $\mathbf{s}^{\alpha}$, while $\rho_{\text {Get }}^{\alpha}$ and $\rho_{\text {Gen }}^{\alpha}$ are edge dislocations and their line tangent vectors are parallel to slip normal $\mathbf{n}^{\alpha}$ and $\mathbf{t}^{\alpha}$ respectively where $\mathbf{t}^{\alpha}=$ $\mathbf{s}^{\alpha} \times \mathbf{n}^{\alpha}$. And $\mathbf{b}^{\alpha}$ is the Burgers vector on slip system $\alpha$.

In order to compute the 36 independent GND components (12 screw and 24 edge dislocation components), the above equation can be expressed as:

$$
\begin{aligned}
& \mathbf{A} \boldsymbol{\rho}_{\mathrm{G}}=\boldsymbol{\Lambda} \\
& \boldsymbol{\rho}_{\mathrm{G}}=\mathbf{A}^{+} \boldsymbol{\Lambda}
\end{aligned}
$$

where $\mathbf{A}$ is a tensor of size of $9 \times 36$ which is formulated in accordance with the methodology developed by Cheng et al [34] and is used to determine $\boldsymbol{\rho}_{\mathbf{G}}$. The matrix of the pseudo-inverse of $\mathbf{A}$ is expressed as $\mathbf{A}^{+}$. Thus, GND density on each individual slip systems [34] is given by

$$
\rho_{\mathrm{GND}}=\sqrt{\rho_{\mathrm{Gs}}^{2}+\rho_{\mathrm{Get}}^{2}+\rho_{\mathrm{Gen}}^{2}}
$$

The initial crystal slip strength and isotropic hardening coefficient $\lambda$ in equations (3), (4) and (5) were determined by simulating polycrystal response under uniaxial tensile test conditions and seeking best agreement with experimental data. Other properties arising in the slip rule have been obtained from the literature (the anisotropic elastic stiffnesses, Burger vector magnitude, Boltzman constant, representative initial mobile dislocation density). Note that the activation energy $\Delta F$ was selected to give negligible rate sensitivity at room temperature. The same crystal slip rule properties are employed for both the single crystal (CMSX4) and oligocrystal (MAR002) studies in this paper. The properties so determined are listed in Table 2 and example predicted polycrystal responses for $\mathrm{R}=-$ 1 and $\mathrm{R}=0$ loading for randomly textured polycrystal are given in Figure 3 . We note that kinematic hardening is not explicitly include in the slip rule, though the evolution of GNDs provides a contribution to kinematic hardening through the establishment of back stresses influencing hardening through equation (4). It is known that kinematic effects are potentially significant in cyclic plasticity, notably in dissipated energy as demonstrated by Morrison et al [35]. However, it has not yet become possible to measure microstructure-level hysteresis behaviour (though high energy x-ray diffraction measurements may provide this in future) with which to demonstrate agreement with crystal plasticity models, so we do not attempt to include these effects in the crystal slip rule. 
Table 2. Material properties for crystal slip in Ni-base alloy CMSX04 and MAR002

\begin{tabular}{c|c|c|c|c|c|c}
\hline $\begin{array}{c}\tau_{c 0} \\
(M P a)\end{array}$ & $\begin{array}{c}b \\
(\mu \mathrm{m})\end{array}$ & $\begin{array}{c}v \\
\left(s^{-1}\right)\end{array}$ & $\begin{array}{c}\lambda \\
\left(\mu m^{-2}\right)\end{array}$ & $\begin{array}{c}k \\
(J / K)\end{array}$ & $\begin{array}{c}\Delta F \\
(N . m)\end{array}$ & $\begin{array}{c}\rho_{\text {ssdm }} \\
\left(\mu m^{-2}\right)\end{array}$ \\
\hline 350 & $3.5072 \mathrm{e}-4$ & $1 \mathrm{e}+11$ & 150 & $1.381 \mathrm{e}-23$ & $4.913 \mathrm{e}-20$ & 5.0 \\
\hline
\end{tabular}
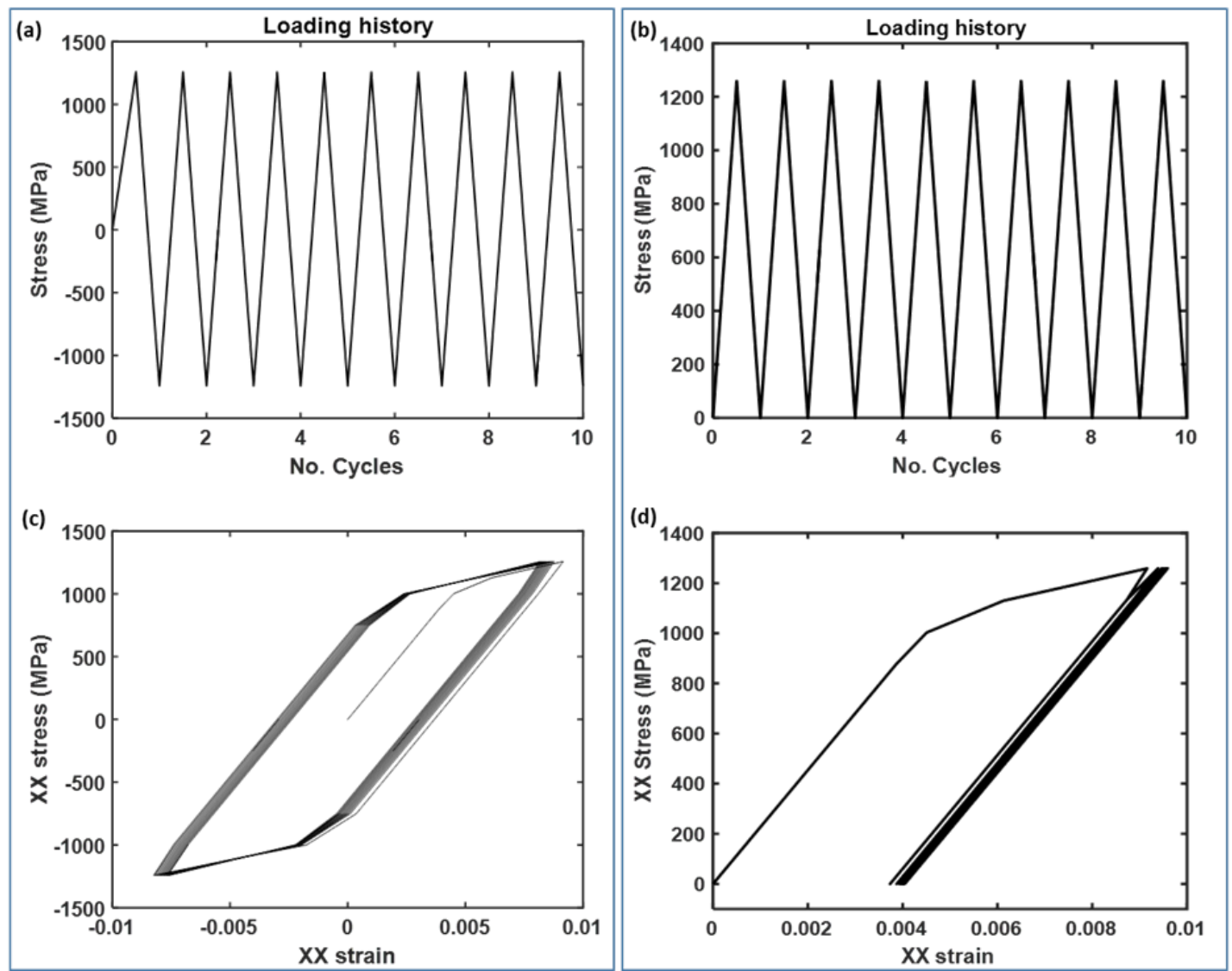

Figure 3. CPFE model predicted stress-strain response to $\mathrm{xx}$-stress-controlled $(\mathrm{R}=-1$ and $\mathrm{R}=0)$ loading of a random polycrystal

\subsubsection{Stored energy density criterion for fatigue crack nucleation}

Many researchers have sought fatigue indicator parameters (FIPs) in polycrystalline materials, and they have been based on a range of quantities including energy, stress, and plastic strain. Accumulated slip, for example, was found to be suitable in one study [36]. However, a range of recent work [4, 14, 27, $29,31,37]$ in nickel and other alloys addressing fatigue crack nucleation utilising high resolution DIC and EBSD has demonstrated that quantities including local stress, accumulated slip (within PSBs), and GND density are necessary but not sufficient indicators of fatigue crack nucleation. While one or more of these quantities is usually associated with the site of defect nucleation, any one of them is not in its own right predictive. Some studies [4, 29] have, however, shown that a local stored energy density (a Griffith-like quantity) does provide reasonable consistency and importantly, is dependent upon and therefore rationalises all the separate quantities sometimes used. In the local criterion of Wan et al. [4, 21], a microstructure-level stored energy is determined and it is argued that a critical value is necessary to be achieved whose value relates to the energy required in order to generate new free surfaces. The local densities of both SSDs and GNDs 
generating a structural contribution to local elastic energy are important and the latter introduces a length scale associated with the defect nucleation. Figure 4 shows schematically a location within a microstructure at which slip localisation occurs, leading to dislocation pile-ups, the development of plastic strain gradients maintained by GND build-up, and the consequent concentration of stress. The resultant local stored energy is high and after a critical number of cycles becomes sufficiently large such that it exceeds that needed to generate new free surfaces over a length scale determined by the density of dislocations.

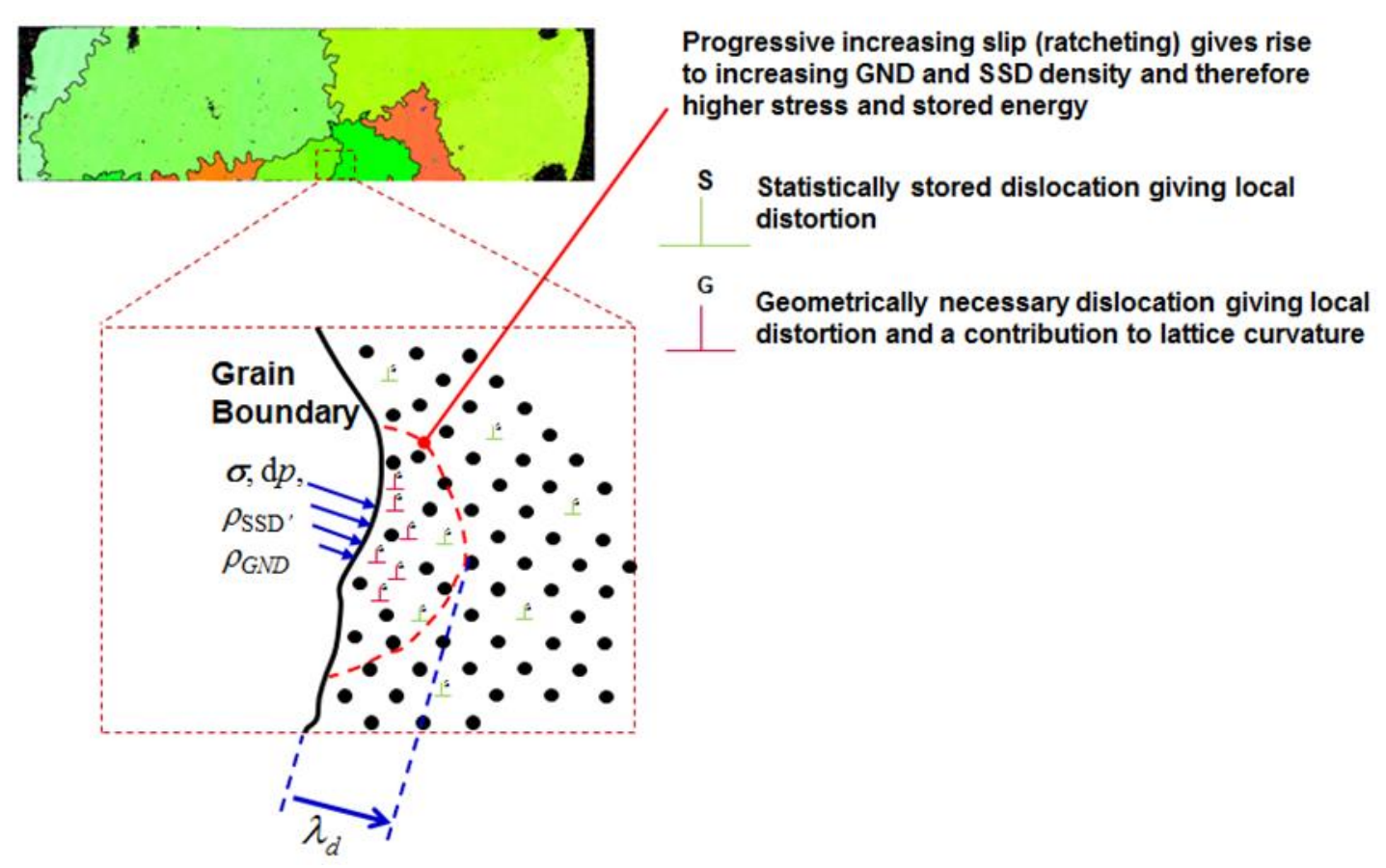

Figure 4. Schematic diagram showing a grain boundary at which persistent slip develops establishing a localised region of high slip, dislocation pile-up, lattice curvature and stress and hence local stored energy over a length scale determined by the dislocation density

To begin, the dissipated energy $\dot{U}\left(\mathrm{~J} / \mathrm{m}^{3}\right)$ per cycle at a microstructural point can be calculated by integrating over a complete loading cycle as

$\dot{U}=\int\left|\boldsymbol{\sigma}: \mathrm{d} \varepsilon^{\mathrm{p}}\right|=\int\left(\left|\sigma_{\mathrm{xx}} \mathrm{d} \varepsilon_{\mathrm{xx}}^{\mathrm{p}}\right|+\left|\sigma_{\mathrm{yy}} \mathrm{d} \varepsilon_{\mathrm{yy}}^{\mathrm{p}}\right|+\left|\sigma_{\mathrm{zz}} \mathrm{d} \varepsilon_{\mathrm{zz}}^{\mathrm{p}}\right|+2\left|\sigma_{\mathrm{xy}} \mathrm{d} \varepsilon_{\mathrm{xy}}^{\mathrm{p}}\right|+2\left|\sigma_{\mathrm{xz}} \mathrm{d} \varepsilon_{\mathrm{xz}}^{\mathrm{p}}\right|+2\left|\sigma_{\mathrm{yz}} \mathrm{d} \varepsilon_{\mathrm{yz}}^{\mathrm{p}}\right|\right)$

As the energy is associated with fatigue crack nucleation, the volume within which energy is stored can be expressed as:

$$
\Delta \mathrm{V}_{\mathrm{s}}=\lambda^{\prime} \Delta \mathrm{A}_{\mathrm{s}}=\frac{\Delta \mathrm{A}_{\mathrm{s}}}{\sqrt{\rho_{\mathrm{SSD}}+\rho_{\mathrm{GND}}}}
$$

where $\Delta \mathrm{V}_{\mathrm{s}}$ is the storage volume, and $\lambda^{\prime}$ is the mean free distance of immobile dislocations. Hence, the stored energy density per cycle $(\dot{G})$ can be written as

$$
\dot{\mathrm{G}}=\frac{\dot{\mathrm{U}} \Delta \mathrm{V}_{\mathrm{s}}}{\Delta \mathrm{A}_{\mathrm{s}}}=\int \frac{\zeta\left|\boldsymbol{\sigma}: \mathrm{d} \varepsilon^{\mathrm{p}}\right|}{\sqrt{\rho_{\mathrm{SSD}}+\rho_{\mathrm{GND}}}}
$$

in which $\zeta$ represents the fraction of the dissipated energy that is stored elastically as dislocation structures within the storage volume, giving the stored energy rate $\dot{\mathrm{G}}$ with units of $\mathrm{J} / \mathrm{m}^{2}$ per cycle.

It is argued that the stored energy increases rapidly in the early fatigue life but that the subsequent rate of change decreases once dislocation structures are formed after the first few cycles. Littlewood et al. [36] have shown this 
phenomenon in their experiments. Therefore, we argue that the stored energy rate per cycle achieves a steady state when the accumulated slip and dislocation evolution rates achieve a steady state. Hence, it is consequently believed that the balance between stored and dissipated energy has, by this stage, also achieved the steady state and for this reason, we argue that the fraction of plastic energy which

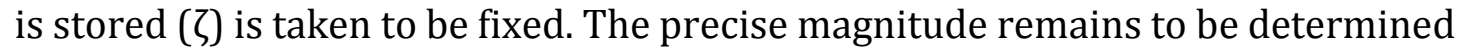
but the often accepted fraction of dissipated energy emerging as heat is well documented to be about $95 \%$, and it is for this reason that we take the fraction of dissipated energy to be stored to be $5 \%$ (i.e. $\zeta=0.05$ ).

The evolution of stored energy, $G$, is therefore written in terms of that accumulated rapidly in the first few cycles (typically 20), $G_{i}$, and that which accumulates subsequently per cycle, $\dot{G}_{s s}$, once the steady state is achieved so that

$$
G=G_{i}+\int \dot{G}_{s s} d N
$$

in which $N$ is the number of steady state cycles. The condition for fatigue crack nucleation is then achieved when the stored energy equates to a critical value, $G_{C}$, thereby giving the number of cycles required to nucleate the crack, $N_{\text {nucleation. }}$. In the CPFE implementation, for every model sample (single or oligocrystal), the stored energy rate is calculated fully coupled within the CP model using equation (13) and is updated (everywhere in the model) at every time integration step in the analysis until a steady state (approximately constant rate of evolution) of stored energy rate $\dot{G}_{s s}$ (energy per cycle) has been achieved. Samples for which the loading was separated in to prior and subsequent regimes (see Table 1) were analysed in exactly the same way. The stored energy at the steady state is labelled $G_{i}$. Subsequent to this, the evolution is simply taken to be given by $\int \dot{G}_{s s} d N$ and $\dot{G}_{s s}$ is the steady state constant stored energy rate and this may be carried out (separate to the full CPFE analysis) over however many cycles is necessary to obtain the cycles to achieve the critical stored energy, $G_{C}$, and hence crack nucleation at any given location.

\subsubsection{Introducing nucleated cracks with XFEM (extended finite element method)}

The extended finite element method (XFEM) is introduced to model cracks that have nucleated at experimentally observed and predicted sites in the Ni single crystal model bend fatigue samples. The XFEM method allows the introduction of arbitrary discontinuities (e.g. cracks) in the finite element displacement field of an element that is enriched with additional displacement field terms but with the standard FE basis functions still used [38-40]. The Abaqus implementation of XFEM utilises the phantom-node formulation proposed by Song et al [41] and the 'propagating crack formulation' in Abaqus does not include crack tip enrichment which makes it effectively identical to a cohesive zones approach for the investigation presented here where the cracks introduced are stationary.

The methodology adopted in this work for crack nucleation in the model single crystal bend fatigue samples is to identify the location of fatigue crack nucleation predicted from the stored energy described in Section 2.3.2, and to introduce an XFEM crack at this location, of length and orientation obtained from the corresponding experimental observations. The consequence of the introduction 
of the nucleated crack is to precipitate the redistribution of all local microstructural quantities including slip, stress, GND density and stored energy. These quantities are then assessed in the context of the nucleation of the experimentally observed secondary cracks, specifically in order to determine if the secondary crack nucleation sites are correctly predicted by the stored energy density. This investigation is described fully in a later section.

\section{Results}

\subsection{Experimental and crystal plasticity model cyclic testing of single and oligocrystal beams}

The regions of interest (ROIs are indicated in Figure 1) on the single and oligocrystal beams tested under cyclic three-point loading are shown in Figure 5 (column 2) to reveal the local microstructures. The detailed crystallographic orientations at ROIs of the oligocrystal samples are shown in Figure 6. The HR-DIC measured and CPFE predicted total xx (bending) strains after 20 fatigue cycles at the ROIs of each sample are given in Figure 5 (column 3 and column 4). Note that differing strain range scale bars are utilised for each sample in order to highlight the strain distributions.

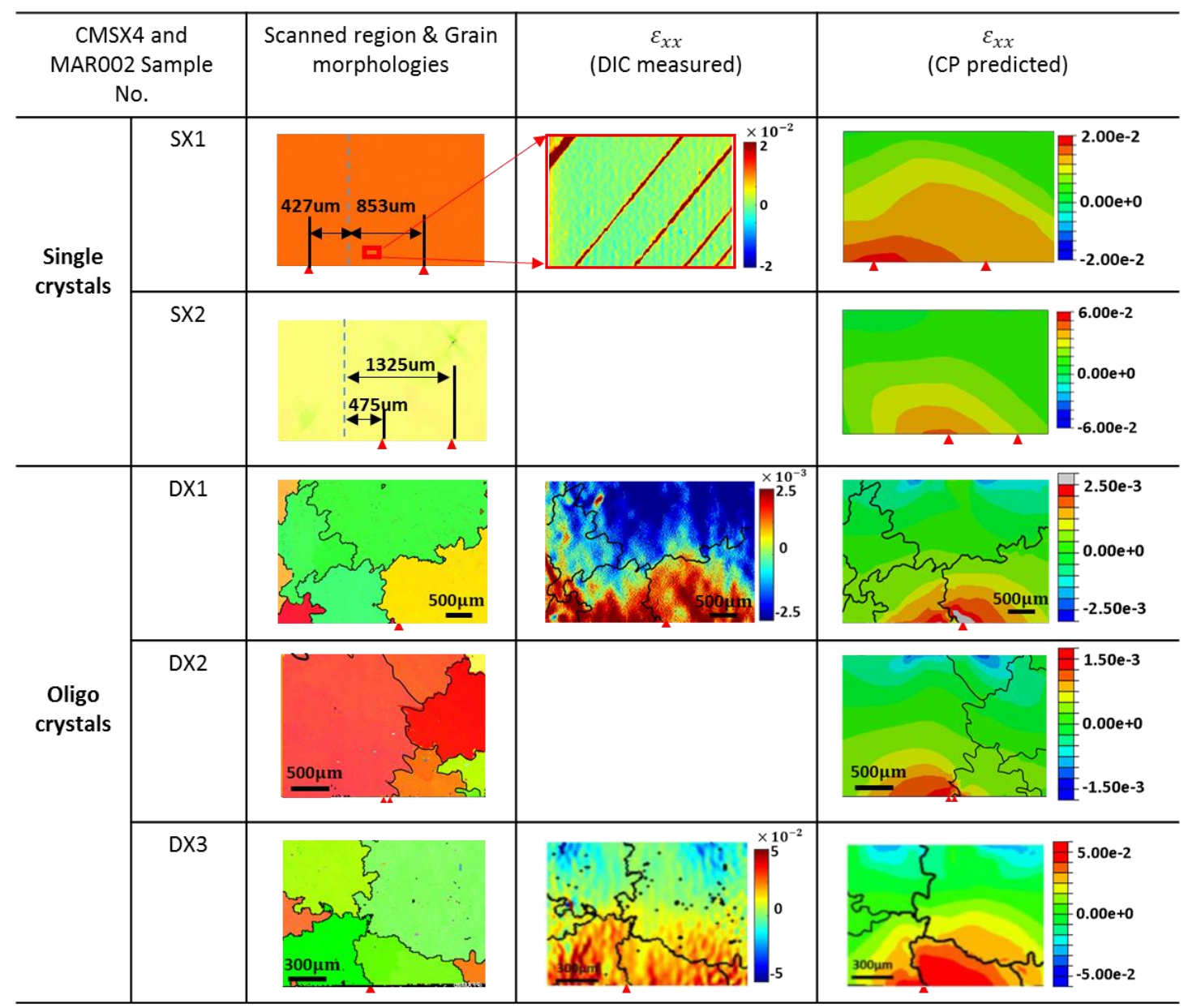

Figure 5. Single crystal and oligocrystal microstructures of the five samples (second column), and DIC measured (third column) and CPFE predicted (fourth column) xx strain at ROIs of the five samples at the end of cycle 20. Red triangles show experimentally observed sites of crack nucleation; black lines represent grain boundaries. 
Crystal plasticity modelling in all cases is 3D, fully representing the samples' morphologies and crystallographies for both single and oligocrystal samples. Details are given in Appendix A.

In the single crystals (SX1 and SX2), the slip initially occurs along discrete, wellseparated slip planes that develop by thickening with subsequent cyclic loading eventually to establish more uniform strain fields, which are shown by the CPFE models (which, of course, are unable to capture the early, highly discrete slip). The strain distributions for both single crystal samples are asymmetric since the crystallographic orientations are such that principal material axes are not coincident with the loading axes. HR-DIC measured and CPFE predicted bending strains are also shown where available for the oligocrystal beams in Figure 5, for which reasonable qualitative comparisons are obtained. Quantitative comparison of experimental and CPFE modelling results of single and multiple slip activation, the distributions of slip and the resulting strain fields in these single and oligocrystals, are the detailed in a previous paper [26] which found excellent quantitative agreement. The focus of this paper, however, is the subsequent fatigue response of the single and oligocrystal beams including the onset of fatigue crack nucleation and microstructurally sensitive growth. For each beam sample, the site or sites of fatigue crack nucleation are shown by the red triangles that were always found to occur at or near the front central bottom base of each sample where the highest tensile bending stress presents. However, within this region, much microstructural sensitivity of fatigue crack nucleation was seen, as shown in Figure 5. For the oligocrystal samples, grain boundaries were found to play a significant role in all cases.

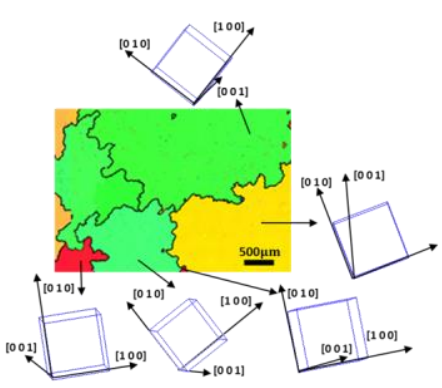

(a) DX1

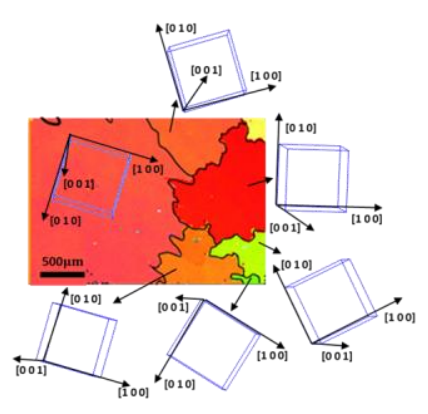

(b) DX2

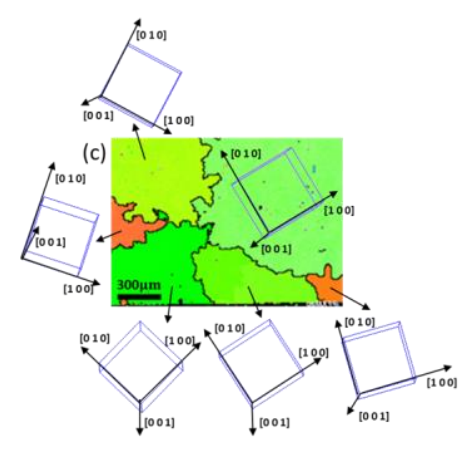

(c) DX3

Figure 6. EBSD measured crystallographic orientations within ROIs for oligocrystals (a) DX1, (b) DX2 and (c) DX3.

\subsection{CPFE assessment of experimental observations}

The results of the CPFE analyses, including accumulated plastic strain and GND density distributions for all beams are shown in Fig. 7. Note that the xx bending stresses shown are at the fully loaded state of the $20^{\text {th }}$ cycle. Full loading histories of samples can be found in table 1. 


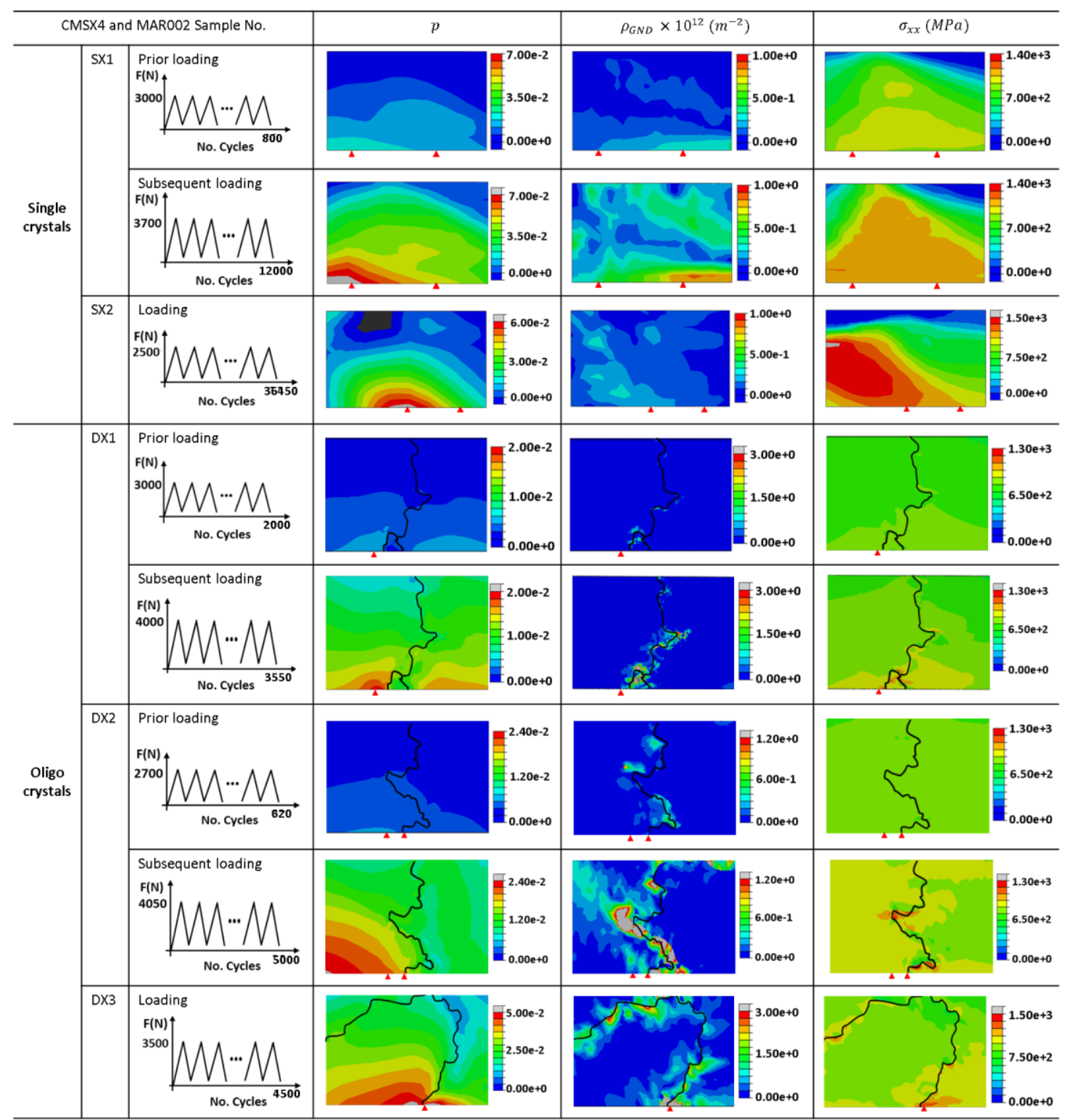

Figure 7. Comparisons of the CPFE predicted distributions of strain, GND density and stress at the ROIs of five nickel-based samples prior and post the load increase (where appropriate).

The results reveal that increasing the peak load in the cycles lead to an increase in magnitude of accumulated plastic strain, GND density and stress without significant change to the nature of the distributions of these quantities. The accumulated plastic strain in single crystals SX1 and SX2 shows quite significant localization. Also, interrogation of the local CPFE results shows that the peak strain attained in the prior loading for sample DX1 is less than $2.0 \%$ but increases to greater than $7 \%$ in the subsequent loading. In oligocrystal DX1, the plastic strain develops within two grains well orientated for slip which straddle a small central grain badly orientated for slip, within which very little plastic strain develops. In sample DX3, high-accumulated plastic strain develops in the large left-side grain and a strong strain gradient can also be seen within this grain. There is also strain localization within the right-side grain over a small region in the vicinity of the grain boundary, but much less slip is predicted at other regions within the same grain. The distributions of GNDs in the oligocrystal samples are almost completely associated with grain boundaries, indicating these are the 
locations where GNDs are necessary in order to accommodate lattice mismatch. In the single crystals, one of the two cracks nucleated (shown in Fig. 7 by the red triangles) for each case coincides with high GND density, and largely, the crack nucleation sites in the oligocrystals are associated with higher GND density, although locations with yet higher GND density exist in the absence of (subsurface) cracking. The experimentally observed fatigue crack nucleation sites are not always found to coincide with locations of highest accumulated plastic strain, or slip, but in fact mostly do so. The exceptions relate to those cases where there is more than one nucleated crack for the two single crystal beams.

The bending stresses in the oligocrystals show perturbations local to the grain boundaries but the only case for which a high stress is unequivocally associated with fatigue crack nucleation site is that for oligocrystal DX3. In summary, therefore, in keeping with other studies [4, 14, 27, 29, 31, 37], it is found that there is an absence of unambiguous correlation between fatigue crack nucleation site and microstructure-level bending stress, GND density or accumulated slip magnitude, although certainly for the oligocrystals (i.e. with grain boundaries), the accumulated slip is a reasonable predictor of crack nucleation site.

\subsection{Fatigue crack observations}

A summary of the three-point bending test results on the single and oligocrystal samples is shown in Fig. 8 (loading histories and fatigue bending test setup can be found in Table 1 and Fig. 1). Two columns are given in which the early stage corresponds to observations as close to crack nucleation as is possible, and the late stage to substantial crack lengths, where cracks are a significant fraction of sample size. The total number of fatigue cycles to give the observed cracks and the corresponding crack length are measured and listed in Fig. 8. The lengths of the first-observed cracks for the different samples, shown in the early stage column in the figure, vary significantly which can be clearly seen in SEM images as shown in Fig. 8 (though crack nucleation was not captured for sample DX1). The locations of each crack with respect to the beam sample front faces may be referenced from Fig. 7 (red triangles). 


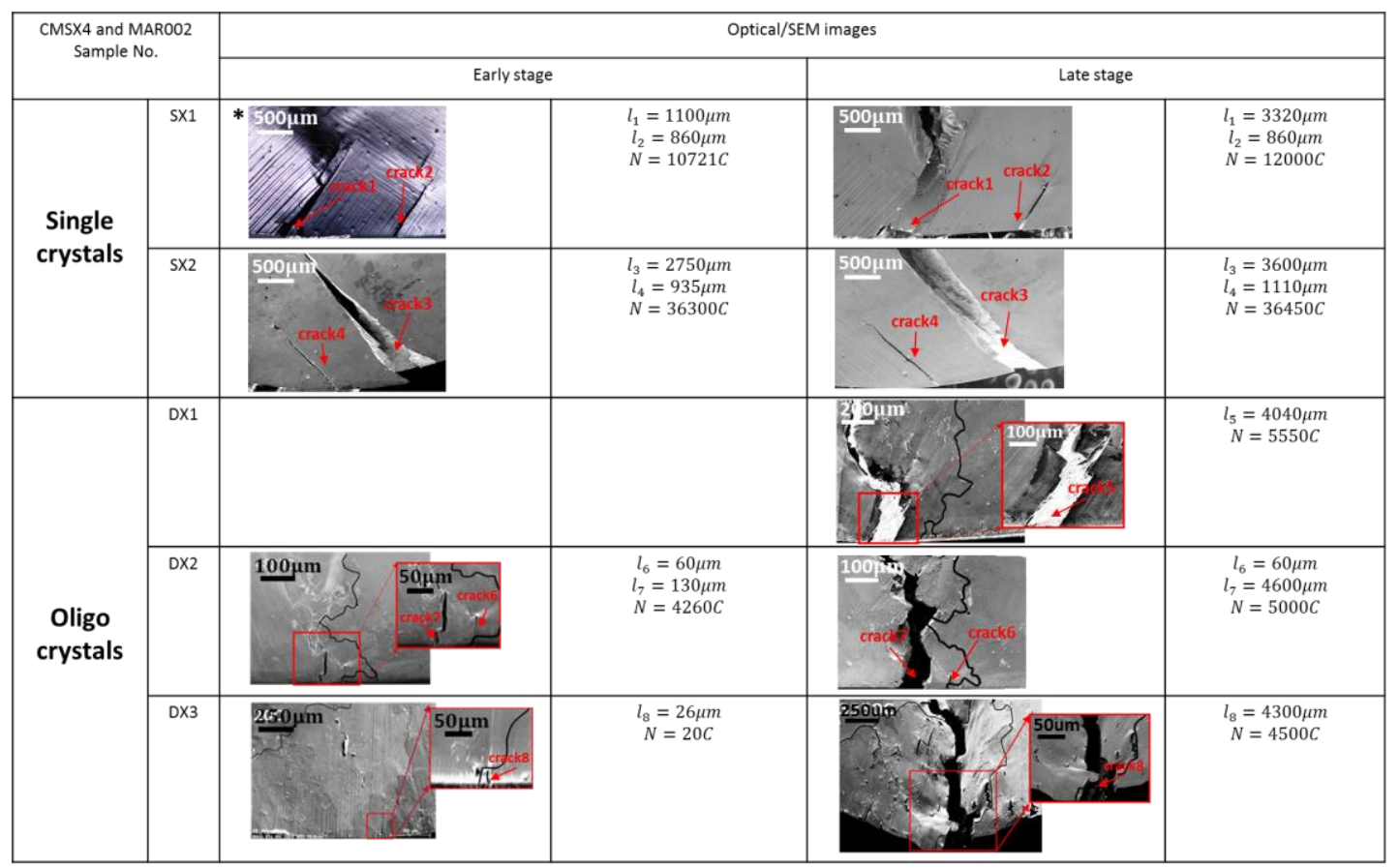

Figure 8. Experimental observations of fatigue crack nucleation sites and growth paths for all the samples. The crack length and number of fatigue cycles are denoted as $l_{i}$ and $\mathrm{N}$ respectively. (*optical image)

In single crystal SX1, from optical and SEM observations, there are two nucleated cracks, labelled crack 1 and crack 2 , which are found at the bottom free surface of the sample. Crack 1 propagates with increasing cycles while crack 2 showed no further growth from 10721 fatigue cycles. The propagation paths are observed to be crystallographic in nature and parallel with slip planes. Similarly, for single crystal sample SX2, two cracks (crack 3 and crack 4) are observed to nucleate from the bottom free surface and then also to grow crystallographically along crystal slip planes. Crack 3 was found to be the more substantial and life-controlling defect in this sample, as was crack 1 for single crystal sample SX1. Both single crystal samples, each with different crystallographic orientation, developed two discrete cracks under fatigue and in both cases, one crack effectively ceases growth to leave the other crack to be that which dominates.

Crack nucleation was not captured for oligocrystal DX1 such that only the fatigue life to fracture was obtained together with the site of crack nucleation. Crack 5 nucleates in the vicinity of a small grain (see Figs. 7 and 8) and then propagates within the large left-side grain until sample failure. The propagation path is much more complex than that of the single crystal cases, and is initially crystallographic following the active slip plane direction until it diverts with significant curvature. In oligocrystal DX2, two cracks (crack 6 and crack 7) nucleate and are captured at lengths of 60 and $130 \mu \mathrm{m}$ respectively in Fig. 8. Crack 6 nucleates on a grain boundary (see Fig. 7 also) whereas crack 7 is very much transgranular. Crack 7 continues to grow rapidly with cycles but crack 6 terminates at a length of $60 \mu \mathrm{m}$ leaving crack 7 to dominate the failure.

In the case of oligocrystal DX3, the presence of a grain boundary seems to play a significant role in fatigue crack nucleation and propagation, again resulting in a more complex fatigue behaviour than in the single crystals. A micro-crack (crack 8 ) is observed to have nucleated close to, but not at a grain boundary, and is shown 
initially at a length of $26 \mu \mathrm{m}$. This crack is short, extending transgranularly. The final crack length is also shown in Fig. 8, but a more detailed evolution of growth of this crack is shown in Fig. 9. Crack 8 nucleates within the first 20 fatigue cycles and then extends to meet a grain boundary after 200 cycles. It remains terminated at the grain boundary for a further 3050 cycles at which point it is seen to traverse the grain boundary. Growth then continues transgranularly until sample failure after a further 1250 cycles.

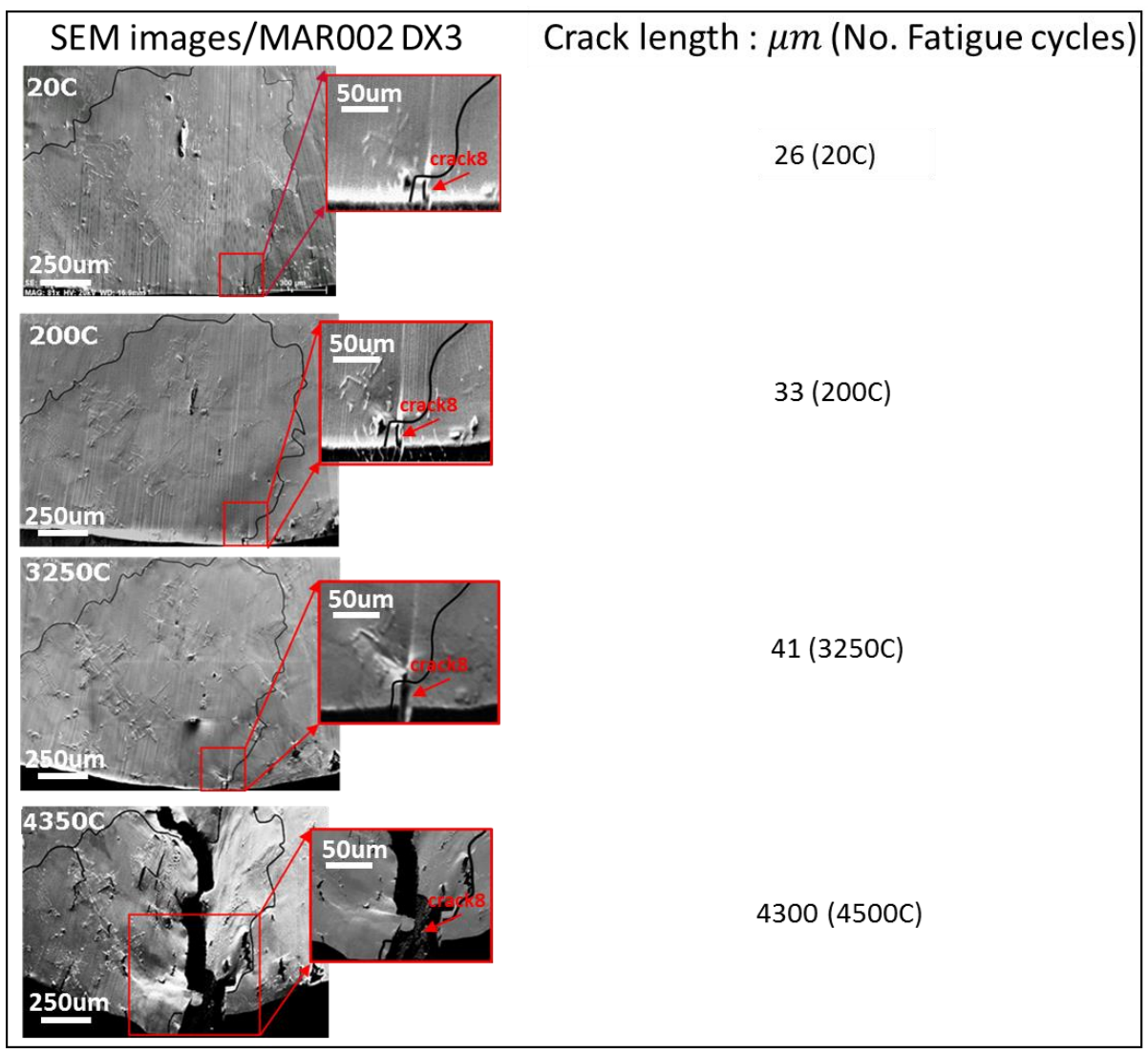

Figure 9. Crack nucleation and propagation in oligocrystal DX3, with recorded crack length and number of fatigue cycles.

For the purposes of subsequent analysis, crack nucleation is defined as when crack length reaches $\sim 1 / 50^{\text {th }}$ of the average grain size, and the latter is measured to be $1220 \mu \mathrm{m}$ in the oligocrystal samples. Hence, crack nucleation is defined to be 25 microns to ensure crack nucleation length is small compared with the grain size. 


\subsection{Assessment of crack nucleation using stored energy density}

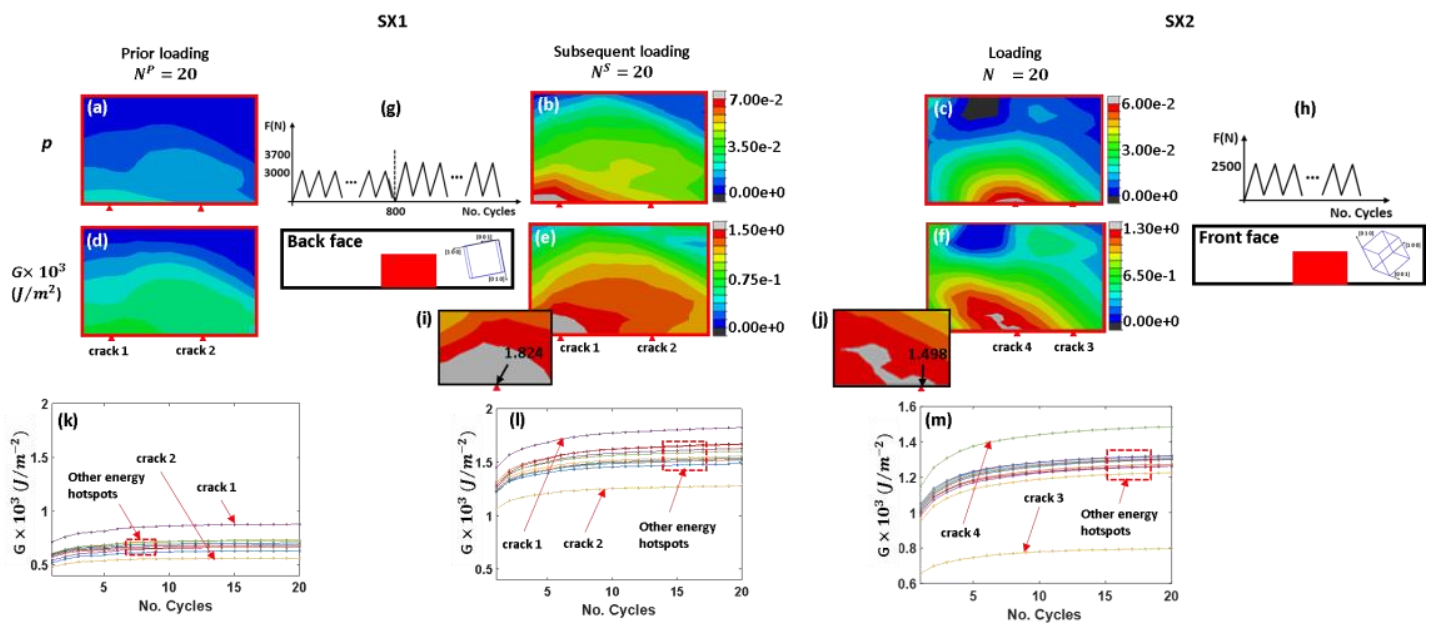

Figure 10. (a)-(c) and (d)-(f)) show the distribution of accumulated plastic strain $p$ and stored energy density $G$, as computed by CPFE, in single crystal SX1 and SX2 respectively. (g) and (h) show loading histories, crystallographic orientations and the mapped ROIs of SX1 and SX2. (i) and (j) are partial enlarged views of $(e)$ and $(f)$ with the location of peak value of stored energy $G$ marked by black arrows. $(k)-(m)$ show the evolution of stored energy over 20 fatigue cycles at key crack and other locations

Fig. 10(a) and (b), (d) and (e) show the accumulated slip and stored energy density after 20 fatigue cycles in single crystal beam SX1 for the prior and subsequent loading respectively. The stored energy density evolution of various 'hotspots' as well as at the locations of cracks 1 and 2 are shown in Fig. 10(k) and (l) for the prior and subsequent loading respectively. It can be clearly seen that the structure and spatial distribution of both accumulated slip and stored energy density remain the same for both the early and subsequent loading stages for sample SX1, and that the key effect of the subsequent load increase is to markedly increase the magnitudes of accumulated slip and stored energy rate. Fig. $10(\mathrm{k})$ and (l) show that in the early stage (lower) loading, the rate of evolution of stored energy stabilises to become very low. The subsequent (higher) loading develops a rapid increase in the magnitude of stored energies and a higher rate of evolution. For sample SX2, the peak load is constant for all 20-fatigue cycles shown in Fig. 10(h). Field plots of the accumulated slip and stored energy density distributions are shown in Fig. 10(c) and (f) respectively, with a zoomed view at first crack nucleation site (crack 4) shown in Fig. 10(j).

The crack nucleation sites for samples SX1 (crack 1) and SX2 (crack 4) are unambiguously demonstrated to occur at locations of highest predicted stored energy, $G$, within regions of high accumulated slip. The locations of cracks 2 and 3 , however, are predicted to have much lower stored energy. Crack 1 is the dominant crack in sample SX1, but it is crack 3 in sample SX2 that dominates in experimental observations. Fig. $10(\mathrm{l})$ and $(\mathrm{m})$ also show the evolution of stored energy at a variety of sites local to the crack locations and demonstrate a clear demarcation of high stored energy at sites of cracks 1 and 4. However, once a crack has nucleated, generating new free surfaces, local energy is released thus changing the local lattice curvatures and stress states, but this cannot yet be captured in the CPFE model.

In order to investigate further the crack nucleation behaviour in the presence of first-generated cracks in both single crystals SX1 and SX2, the extended finite 
element method (X-FEM) is used to introduce cracks at the predicted crack sites for cracks 1 and 4 in Fig. 10, so that the resulting redistribution of stress, slip accumulation, GND density and stored energy may be re-assessed to take account of the nucleation of the cracks and the consequences for secondary crack nucleation. Consider single crystal SX1 first.

It can be seen from Fig. 10 that for single crystal SX1, crack 1 is predicted to nucleate first. Hence, an initial small and stationary (X-FEM) crack of length $240 \mu \mathrm{m}$ at the location of crack 1 is introduced into the model sample where crack nucleation is both predicted (Fig. 10) and observed from the optical image (Fig. 8). The resulting accumulated slip and stored energy distributions that are predicted to develop after a further cycle of loading are shown in Fig. 11. The slip and stored energy fields at the crack tip (crack 1) are highly concentrated but away from the tip, and closer to the sample free surface, are now considerably lower than has been established at the location of the experimentally observed crack 2 . Hence, the nucleation of crack 1 has led to the redistribution of slip, stress and dislocation density such that the distribution of stored energy has also changed considerably, as shown in Fig. 11(c). In the presence of crack 1, the new free-surface location of peak stored energy transpires to occur precisely where crack 2 is experimentally observed to nucleate, and this is shown by the line graph of stored energy along the sample free surface A-A' in Fig. 11(d).

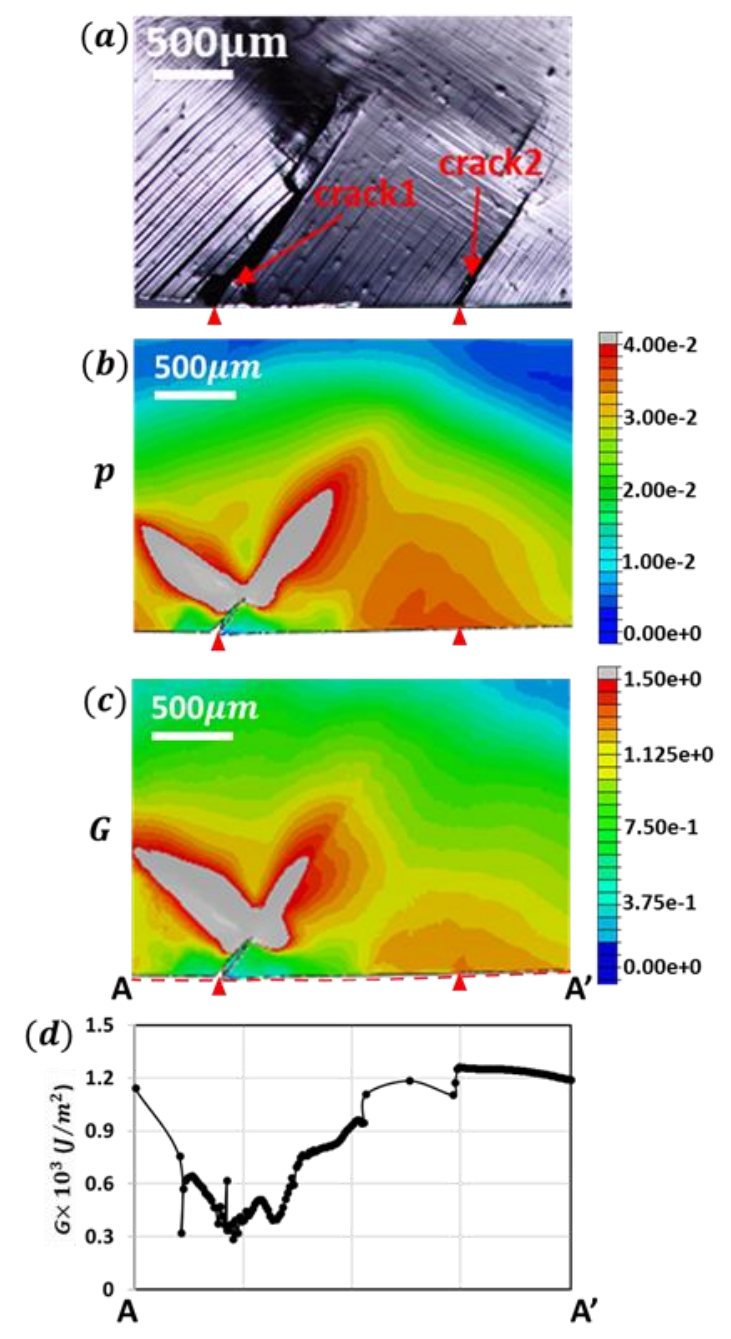


Figure 11. Sample SX1 showing (a) SEM image of ROI with crack 1 and crack 2 indicated, (b) accumulated plastic strain distribution, (c) stored energy density distribution within the ROI, and (d) line graph of stored energy density along sample free surface $A-A^{\prime}$.

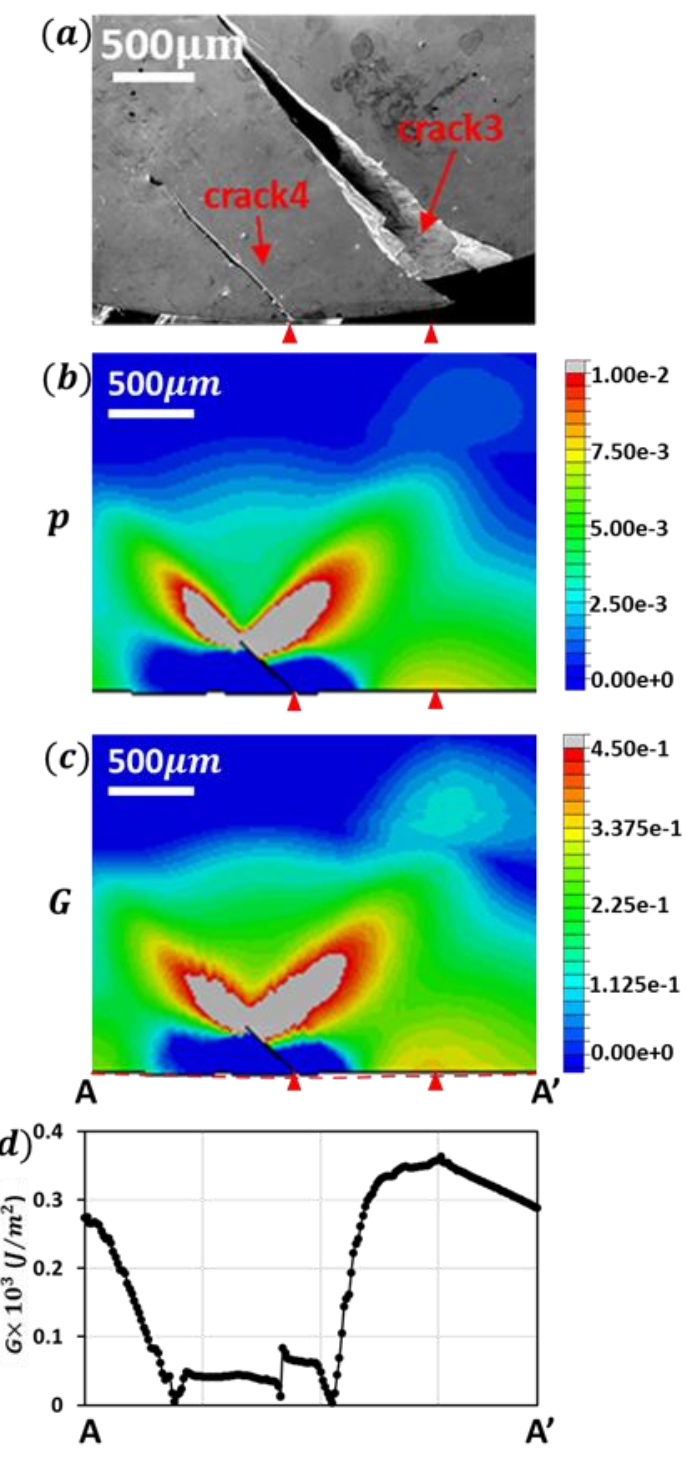

Figure 12. Sample SX2 showing (a) optical image of the ROI with crack 3 and crack 4 indicated, (b) accumulated plastic strain distribution over the ROI, (c) stored energy density distribution within the $\mathrm{ROI}$, and (d) line graph of stored energy density along sample free surface $A-A^{\prime}$.

Similarly, the same method has been utilized to analyse the second crack nucleation behaviour in single crystal SX2. In this case, a crack $(400 \mu \mathrm{m})$ is introduced at the location of crack 4 since it is at this location where crack nucleation is first predicted to occur. As before, the loading has then been continued for a further full cycle and the new distributions of slip accumulation, and stored energy obtained as shown in Fig. 12.

In a similar way for sample SX1, the re-distributed stored energy in the presence of crack 4 in sample SX2 leads to the highest stored energy occurring at precisely that location where crack 3 is experimentally observed to nucleate and subsequently grow. Hence, it is argued that the stored energy density distributions successfully identify locations of primary crack nucleation, and when account is taken of the presence of the newly nucleated cracks, the resulting 
redistributions of stored energy indicate where secondary crack nucleation is expected to develop. So far as the authors are aware, this is the only demonstrable quantitative prediction of primary and secondary fatigue crack nucleation sites observed in experiments. We next consider the oligocrystals DX1, DX2 and DX3.

CPFE predicted accumulated slip and stored energy density results for the three oligocrystal cyclic loading tests are shown in Fig. 13, together with the experimentally observed crack nucleation sites (again shown by red triangles). Crack nucleation is seen to be associated with free-surface grain boundaries in samples DX2 (crack 6) and DX3 (crack 8) but not so for samples DX1 (crack 5) and DX2 (crack 7). Hence both intergranular and transgranular crack nucleation are observed experimentally, with both occurring in sample DX2. Crack 5 in oligocrystal beam DX1 nucleates at a strain accumulation hot spot, as shown in Fig. 13(g). However, this is not the case for oligocrystals DX2 and DX3 shown in Fig. 13(h) and (i) for which the crack nucleation sites do not coincide with peak slip accumulation. For crack 8 in sample DX3, the crack nucleates at a grain boundary in between two regions of high slip.
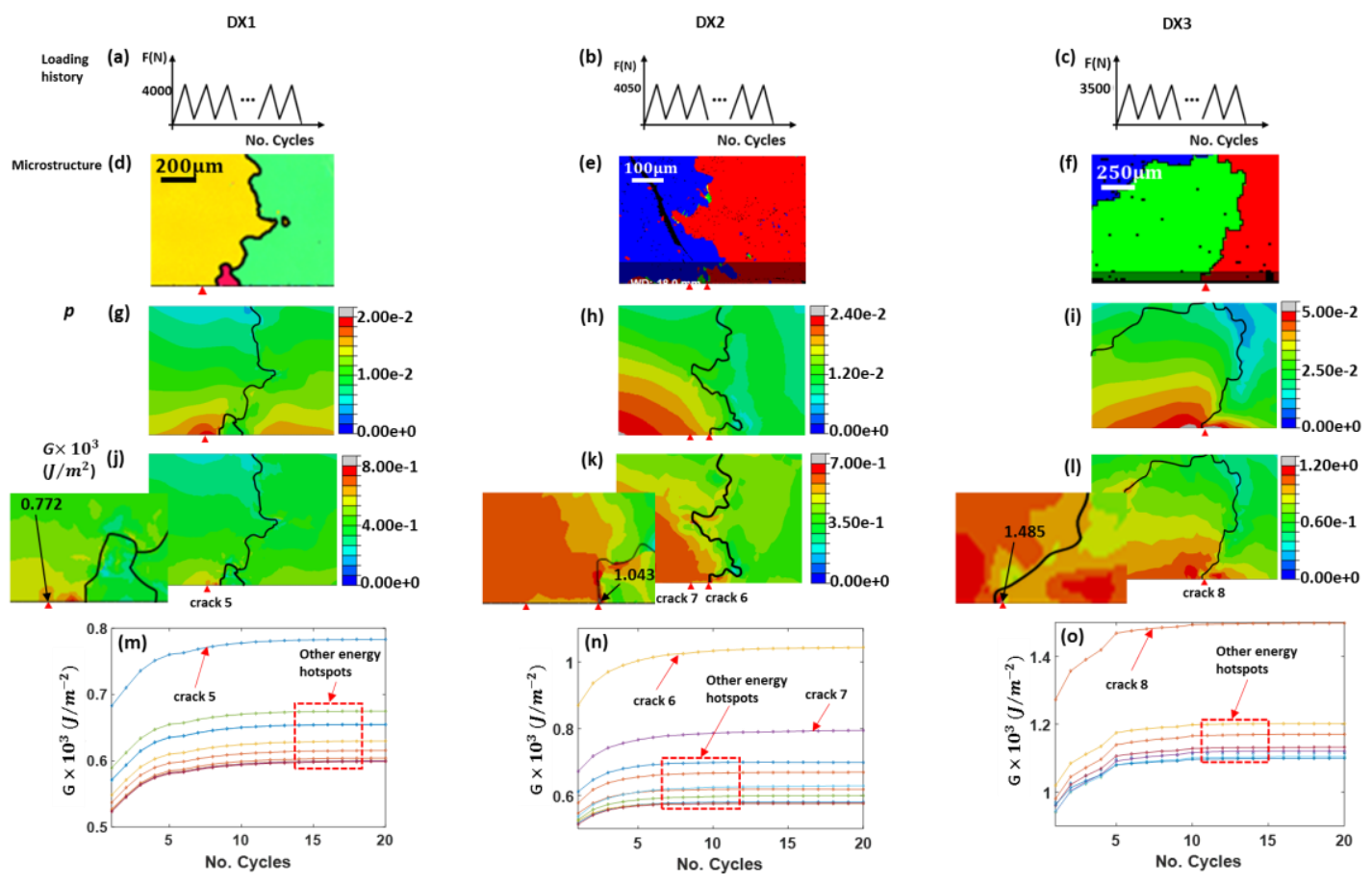

Figure 13. The subsequent loading histories for oligocrystal samples DX1, DX2 and DX3 are shown in (a) - (c) respectively, with the associated local ROI morphology of each sample given in (d) - (f). (g) - (i) show the CPFE predicted distributions of accumulated plastic strain while (j) - (I) show the corresponding stored energy density distributions after 20 loading cycles, with a partial enlarged views shown in the left corner of (j) - (I) respectively showing peak values of stored energy density identified by black arrows. The evolution of stored energy $G$ over 20 loading cycles at the experimentally observed sites of crack nucleation (red triangular symbols), and other local hotspots, is shown in (m) - (o).

The stored energy density, determined from local accumulated slip, GND and SSD density, and stress proposed in [4] is shown in Figs. 13(j), (k) and (l). Peak values of stored energy are found to occur at all of the observed crack nucleation sites in the oligocrystal beams, whether intergranular or transgranular, and unambiguously demarcates the crack nucleation sites in all samples with the possible exception of crack 7 in sample DX2. However, the stored energies at all the sites of crack nucleation and at other sites are shown in the graphs in Figs. 
13(m), (n) and (o). For sample DX2 and cracks 6 and 7, the stored energies at the crack nucleation sites and between these two locations are shown in Fig. 13(n) where it is apparent that the stored energies at cracks 6 and 7 remain considerably higher than at locations in between. The peak stored energy values for samples DX1 and DX3 at cracks 5 and 8 shown in Fig. 13(m) and (o) are markedly higher than at other locations in the vicinity.

\subsection{Predicted fatigue crack nucleation life}

In the case of oligocrystal beam DX3, the nucleated crack length (see Fig. 9) is measured to be $26 \mu \mathrm{m}$ after 20 cycles of loading, and this is defined to be crack nucleation. The critical stored energy density corresponding to this crack nucleation is therefore extracted using the crystal plasticity model for this sample and found to be $G_{c}=1,498 \mathrm{~J} / \mathrm{m}^{2}$. In this work, this critical value, together with CPFE predicted stored energy density rate $\dot{G}\left(\mathrm{~J} / \mathrm{m}^{2}\right.$ per cycle) at each experimentally observed crack nucleation site (Figs. 7 and 8) are used to calculate the required number of cycles for crack nucleation utilising equation (14) in the other oligocrystal samples (DX1, DX2)

Predicted cycles to fatigue crack nucleation for the single crystal beams (SC1 and SC2) transpire to be short, and this is reflected in the experiments also where cycles to crack nucleation (i.e. a crack length less than $25 \mu \mathrm{m}$ ) were difficult to capture experimentally. The cycles to crack nucleation in the oligocrystal beams, however, were typically much (two to three orders of magnitude) higher with the exception of oligocrystal DX3. Predicted cycles to nucleation together with experimentally observed cycles to beam sample failure are shown together for discussion in Fig. 14. However, the key point is that the crack nucleation process is found to be strongly microstructurally sensitive both in the oligocrystal experiments, and as captured by the crystal plasticity model. The figure makes clear the experimentally verified role of microstructure sensitivity in fatigue; higher cyclic loads would otherwise be anticipated to lead to lower cycles to nucleation and failure, but clearly this is not found to be so in the oligocrystal samples.

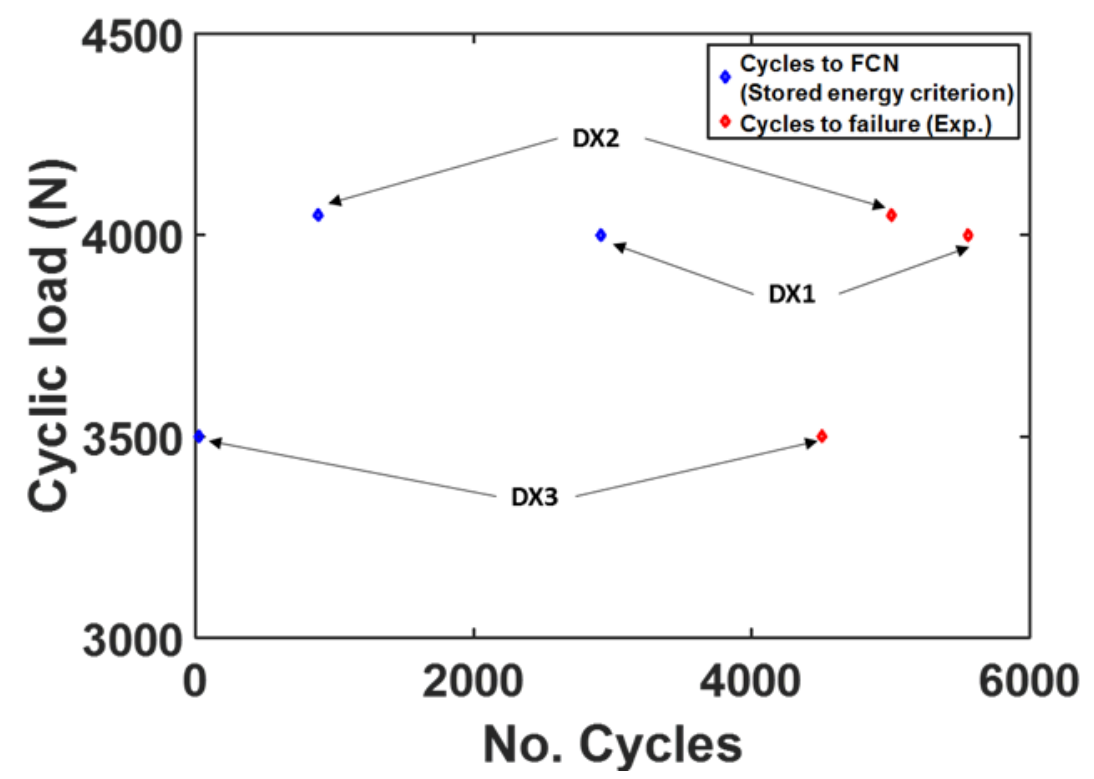


Figure 14. Applied cyclic loading vs number of fatigue cycles to predicted crack nucleation and experimentally observed sample failure for the oligocrystal beam samples

\section{Discussion}

Fatigue crack nucleation and microstructurally sensitive crack growth are complex processes that in many ways continue to elude quantitative prediction, largely because of the absence of mechanistic understanding. Substantial progress has been made recently, achieved by bringing to bear advanced microscopy and characterisation techniques (HR-EBSD, HR-DIC) combined with high fidelity microstructure-level modelling. This paper addresses the mechanistic basis of fatigue crack nucleation by considering simple, model nickel alloy systems in single and oligocrystal form. This methodology facilitates quantitative surface characterisation which is representative of sub-surface behaviour and which provides the ability to utilise dislocation-based crystal plasticity modelling to examine in detail the quantitative mechanics directly at the locations where fatigue cracks are observed to nucleate and grow. It allows full and direct representation of some of the microstructural features argued to be important in fatigue crack nucleation and by bringing together the characterisation, measurement and modelling methods, provides interesting insights in to the quantitative mechanistic basis of fatigue crack nucleation in engineering alloys.

A growing range of experimental studies indicates the relevance of localisation and accumulation of slip in fatigue crack nucleation [4, 7, 14, 27, 29, 31, 37, 42]. Studies have also suggested the distribution of geometrically necessary dislocations may be important [32, 43, 44], along with the local stress distributions [45-48]. GND coupling with slip hardening provides better prediction of lattice rotations and distributions of local stress and strain, and incorporates the length scale in CPFE modelling [43]. It can be seen from the field plots of GND density (shown in Fig. 7) that the grain boundaries play an important role in the dislocation pileup in the oligocrystal samples. For example, the GND density in the single crystals computed by the CPFE models is of order $\sim 1 \times 10^{12} \mathrm{~m}^{-2}$, which is a little smaller than that found for the oligocrystals $\left(\sim 3 \times 10^{12} \mathrm{~m}^{-2}\right)$. The differences in GND densities for each beam result from the differing microstructures and local crystallographic constraints. The presence of grain boundaries, twins, and any irregular geometry (for example, oxide agglomerates or second phases) may play a role in dislocation pile-up. For instance, the average GND density at the interface of a carbide and surrounding nickel matrix is $\sim 3 \times 10^{14} \mathrm{~m}^{-2}$ [44]. GND densities of $\sim 10^{14} \mathrm{~m}^{-2}$ have also been observed in Ni polycrystals containing non-metallic inclusions [32]. Hence, it is argued that high densities of GNDs can be found at locations generating inhomogeneity such as triple junctions, twin boundaries, and inclusions where significant lattice rotations and strain gradients are expected. Nevertheless, locations of high GND density do not always show direct correlation with the locations of fatigue crack nucleation for most of the samples studies in the present work. There are many experimental results showing the potential importance of GND density and other quantities but also that they do not, in their own right, provide the mechanistic basis nor unambiguous prediction of either crack nucleation site nor of the number of cycles necessary to cause nucleation $[4,14]$. This paper has focused particularly on the sites of fatigue crack nucleation, recognising that the latter is a process dominated by microstructure, thereby 
showing marked microstructural sensitivity. Understanding microstructural sensitivity of the site of fatigue crack nucleation must surely be crucial to quantitative mechanistic understanding and is an absolute prerequisite to understanding subsequent growth rate and crack path. Any model for fatigue that fails to identify firstly where in a microstructure fatigue cracks nucleate, it is argued, cannot be strongly mechanistic.

This paper, and several preceding it, has assessed slip accumulation and localisation, local stress, and GND density distributions in fatigue crack nucleation and in this study demonstrated evidence that these quantities are necessary but not sufficient in their own right to drive nucleation. A stored energy density, determined at the appropriate microstructural length scale, and which crucially depends on the above quantities, has in the current work been shown to unambiguously identify the locations of experimentally observed crack nucleation in single and oligocrystals in cyclic plasticity and fatigue. Importantly, it has also been shown that once primary fatigue cracks nucleate, redistribution of key quantities (such as stress, GND density, accumulating slip) occurs thus leading to new locations of peak stored energy such that secondary crack nucleation may occur. The mechanistic basis argued is that a local (elastic) stored energy density, developed through slip localisation, dislocation pile-up and lattice curvature formation by GNDs, resulting in locally high stresses, is established. With further cycling, it increases (in the absence of relaxation and recovery processes) until such time that a critical value, $G_{c}$, is achieved which is equal to that necessary to generate new free surfaces; i.e. to nucleate a fatigue crack. This mechanism has been demonstrated quantitatively to predict the sites of fatigue crack nucleation for cracks in single crystals where the strain inhomogeneity develops because of the non-coincidence of the principal material axes with loading direction. In the presence of grain boundaries (and other important microstructural features such as triple junctions, twins and second phases of an appropriate length scale), the key drivers for heterogeneity are different and stronger than those for the single crystal case and result from the local elastic strain incompatibilities at grain boundaries, the farther field stresses developed by the crystallographic elastic anisotropies, and importantly the plastic anisotropies defined by the local crystallography. Previous studies have examined the latter effects in some detail [26]. It is for these reasons that the stored energy density captures fatigue crack nucleation sites in oligocrystal beam samples which are both transgranular (DX1 and DX3) and intergranular (DX2) in nature, as shown in Fig. 13, reflecting the differing microstructural drivers for local heterogeneity, in turn driving high stored energy and crack nucleation.

\section{Conclusion}

In this study, three-point bending tests, HR-DIC measurements and microstructure-representative CPFE together with X-FEM crack modelling have been carried out to provide insight in to the quantitative mechanistic basis of fatigue crack nucleation in model nickel-base superalloys, including single crystal CMSX04 and oligocrystal MAR002. The experimentally observed fatigue crack nucleation sites have enabled a focused assessment of key microstructural quantities which arguably drive crack nucleation, namely accumulated slip, stress, and GND density. 
The key conclusion is that no one of these quantities alone establishes the fatigue crack nucleation site unambiguously. It is, however, argued that they are necessary but not sufficient in their own rights to drive crack nucleation. The quantity which does identify all fatigue crack nucleation sites in the single and polycrystal samples analysed is the local (elastic) stored energy density, measured over a length scale defined by the dislocation density. In addition, when primary fatigue cracks which are predicted and observed to nucleate in the single crystal samples are introduced in to the crystal models, the secondary fatigue crack nucleation sites observed experimentally are also predicted. The key role of microstructure (here crystallography, grain boundaries and constraint) is to determine the localisation of slip, stress and GND density which drive local stored energy, which in turn leads to fatigue crack nucleation.

\section{Acknowledgments}

$\mathrm{BC}$ is grateful for the financial support provided by Imperial College-China Scholarship Council (IC-CSC), and FPED for Royal Academy of Engineering/RollsRoyce RAEng Research Chair funding. The authors also acknowledge the Beijing Institute of Aeronautical Materials (BIAM), and express thanks to Dr Ben Britton for helpful discussions on the experiments.

\section{Appendix A Single and Oligocrystal Model Sample Finite Element Representations and Mesh Refinements}

\begin{tabular}{|c|c|c|c|c|}
\hline & & Microstructural sensitive CPFE models & Front surface & Refined mesh regions \\
\hline \multirow{2}{*}{$\begin{array}{l}\text { Single } \\
\text { crystals }\end{array}$} & sx1 & $\infty d^{\text {It }}$ & & \\
\hline & $5 \times 2$ & $\sigma$ & & \\
\hline \multirow{3}{*}{$\begin{array}{l}\text { Oligo } \\
\text { crystals }\end{array}$} & DX1 & & & \\
\hline & $\mathrm{DX} 2$ & $-\infty$ & & \\
\hline & DX3 & 2 & & \\
\hline
\end{tabular}




\section{References}

1. M. Fried, et al. Fatigue crack initiation in nickel-based superalloys studied by microstructure-based FE modeling and scanning electron microscopy. in MATEC Web of Conferences. 2014. EDP Sciences.

2. F. Farukh, et al., Fatigue crack growth in a nickel-based superalloy at elevated temperature-experimental studies, viscoplasticity modelling and XFEM predictions. Mechanics of advanced materials and modern processes, 2015. 1(1): p. 1.

3. T. Zhai, A. Wilkinson, and J. Martin, A crystallographic mechanism for fatigue crack propagation through grain boundaries. Acta materialia, 2000. 48(20): p. 4917-4927.

4. V. Wan, D. MacLachlan, and F.P.E. Dunne, A stored energy criterion for fatigue crack nucleation in polycrystals. International Journal of Fatigue, 2014. 68: p. 90-102.

5. J. Polák and J. Man, Experimental evidence and physical models of fatigue crack initiation. International Journal of Fatigue, 2016.

6. K. Miller and M. Ibrahim, Damage accumulation during initiation and short crack growth regimes. Fatigue \& Fracture of Engineering Materials \& Structures, 1981. 4(3): p. 263-277.

7. F.P.E. Dunne, A. Wilkinson, and R. Allen, Experimental and computational studies of low cycle fatigue crack nucleation in a polycrystal. International Journal of Plasticity, 2007. 23(2): p. 273-295.

8. C.A. Stein, et al., Fatigue crack initiation, slip localization and twin boundaries in a nickel-based superalloy. Current Opinion in Solid State and Materials Science, 2014. 18(4): p. 244-252.

9. M.D. Sangid, H.J. Maier, and H. Sehitoglu, A physically based fatigue model for prediction of crack initiation from persistent slip bands in polycrystals. Acta Materialia, 2011. 59(1): p. 328-341.

10. S.R. Yeratapally, et al., Microstructure based fatigue life prediction framework for polycrystalline nickel-base superalloys with emphasis on the role played by twin boundaries in crack initiation. Acta Materialia, 2016. 107: p. 152-167.

11. A.M. Korsunsky, et al., Comparative assessment of dissipated energy and other fatigue criteria. International Journal of Fatigue, 2007. 29(9): p. 1990-1995.

12. M. Oja, K.R. Chandran, and R. Tryon, Orientation Imaging Microscopy of fatigue crack formation in Waspaloy: Crystallographic conditions for crack nucleation. International Journal of Fatigue, 2010. 32(3): p. 551-556.

13. J. Stinville, et al., A combined grain scale elastic-plastic criterion for identification of fatigue crack initiation sites in a twin containing polycrystalline nickel-base superalloy. Acta Materialia, 2016. 103: p. 461473.

14. C. Sweeney, et al., The role of elastic anisotropy, length scale and crystallographic slip in fatigue crack nucleation. Journal of the Mechanics and Physics of Solids, 2013. 61(5): p. 1224-1240.

15. W.D. Musinski and D.L. McDowell, Simulating the effect of grain boundaries on microstructurally small fatigue crack growth from a focused ion beam notch through a three-dimensional array of grains. Acta Materialia, 2016. 112: p. 20-39. 
16. J. Pang, et al., Inhomogeneous deformation behavior in intercrystalline regions in polycrystalline Ni. Acta Materialia, 2014. 65: p. 393-399.

17. R. Barabash, et al., Twin boundary-induced intrinsic strengthening in Ni. Thin Solid Films, 2013. 530: p. 14-19.

18. J. Miao, T.M. Pollock, and J.W. Jones, Microstructural extremes and the transition from fatigue crack initiation to small crack growth in a polycrystalline nickel-base superalloy. Acta Materialia, 2012.60(6): p. 28402854.

19. T. Gabb, et al., Fatigue resistance of the grain size transition zone in a dual microstructure superalloy disk. International Journal of Fatigue, 2011. 33(3): p. 414-426.

20. J. Pang, R. Rogge, and R. Donaberger, Effects of grain size on intergranular strain evolution in Ni. Materials Science and Engineering: A, 2006. 437(1): p. 21-25.

21. V. Wan, et al., Microstructure-sensitive fatigue crack nucleation in a polycrystalline Ni superalloy. International Journal of Fatigue, 2016. 90: p. 181-190.

22. T. Bieler, et al., Grain boundaries and interfaces in slip transfer. Current Opinion in Solid State and Materials Science, 2014. 18(4): p. 212-226.

23. C. Szczepanski, et al., Demonstration of an in situ microscale fatigue testing technique on a titanium alloy. International Journal of Fatigue, 2013. 57: p. 131-139.

24. $\mathrm{P} . \mathrm{Mu}$, et al., Influence of the crystalline orientations on microcrack initiation in low-cycle fatigue. Materials Science and Engineering: A, 2013. 573: p. 4553.

25. G.M. Castelluccio, W.D. Musinski, and D.L. McDowell, Recent developments in assessing microstructure-sensitive early stage fatigue of polycrystals. Current Opinion in Solid State and Materials Science, 2014. 18(4): p. 180187.

26. Y. Guan, et al., Crystal plasticity modelling and HR-DIC measurement of slip activation and strain localisation in single and oligo-crystal Ni alloys under fatigue. International Journal of Plasticity, 2016. 88: p. 70-88.

27. T. Zhang, et al. Crack nucleation using combined crystal plasticity modelling, high-resolution digital image correlation and high-resolution electron backscatter diffraction in a superalloy containing non-metallic inclusions under fatigue. Proc. R. Soc. Lond. 2016. 472(2189): (DOI: 10.1098/rspa.2016.0037).

28. M. Bornert, et al., Digital image correlation. Full-Field Measurements and Identification in Solid Mechanics, 2012: p. 157-190.

29. J. Jiang, et al., Microstructurally sensitive crack nucleation around inclusions in powder metallurgy nickel-based superalloys. Acta Materialia, 2016. 117: p. 333-344.

30. J. Jiang, et al. Deformation compatibility in a single crystalline Ni superalloy. Proc. R. Soc. A, Vol. 2016. 47:(2185): (DOI: 10.1098/rspa.2015.0690).

31. T. Zhang, et al., Slip localization and fatigue crack nucleation near a nonmetallic inclusion in polycrystalline nickel-based superalloy. Materials Science and Engineering: A, 2015. 641: p. 328-339. 
32. T. Zhang, et al., Crystal plasticity and high-resolution electron backscatter diffraction analysis of full-field polycrystal $\mathrm{Ni}$ superalloy strains and rotations under thermal loading. Acta Materialia, 2014. 80: p. 25-38.

33. H. Gao and Y. Huang, Geometrically necessary dislocation and size-dependent plasticity. Scripta Materialia, 2003. 48(2): p. 113-118.

34. J. Cheng and S. Ghosh, A crystal plasticity FE model for deformation with twin nucleation in magnesium alloys. International Journal of Plasticity, 2015. 67: p. 148-170.

35. J. Moosbrugger and D. Morrison, Nonlinear kinematic hardening rule parameters-direct determination from completely reversed proportional cycling. International journal of plasticity, 1997. 13(6-7): p. 633-668.

36. P. Littlewood and A. Wilkinson, Local deformation patterns in Ti-6Al-4V under tensile, fatigue and dwell fatigue loading. International Journal of Fatigue, 2012. 43: p. 111-119.

37. J. Jiang, et al., On the mechanistic basis of fatigue crack nucleation in $\mathrm{Ni}$ superalloy containing inclusions using high resolution electron backscatter diffraction. Acta Materialia, 2015. 97: p. 367-379.

38. T. Belytschko and T. Black, Elastic crack growth in finite elements with minimal remeshing. International journal for numerical methods in engineering, 1999. 45(5): p. 601-620.

39. T. Belytschko, et al., Arbitrary discontinuities in finite elements. International Journal for Numerical Methods in Engineering, 2001. 50(4): p. 993-1013.

40. J. Dolbow and T. Belytschko, A finite element method for crack growth without remeshing. International journal for numerical methods in engineering, 1999. 46(1): p. 131-150.

41. J.H. Song, P. Areias, and T. Belytschko, A method for dynamic crack and shear band propagation with phantom nodes. International Journal for Numerical Methods in Engineering, 2006. 67(6): p. 868-893.

42. M. Pham and S. Holdsworth, Role of microstructural condition on fatigue damage development of AISI $316 \mathrm{~L}$ at 20 and $300 \mathrm{C}$. International Journal of Fatigue, 2013. 51: p. 36-48.

43. F.P.E. Dunne, R. Kiwanuka, and A. Wilkinson, Crystal plasticity analysis of micro-deformation, lattice rotation and geometrically necessary dislocation density. Proceedings of the Royal Society A: Mathematical, Physical and Engineering Science, 2012. 468(2145): p. 2509-2531.

44. P.S. Karamched and A.J. Wilkinson, High resolution electron back-scatter diffraction analysis of thermally and mechanically induced strains near carbide inclusions in a superalloy. Acta Materialia, 2011. 59(1): p. 263-272.

45. P. Shanthraj and M. Zikry, Microstructurally induced fracture nucleation and propagation in martensitic steels. Journal of the Mechanics and Physics of Solids, 2013. 61(4): p. 1091-1105.

46. M.H. Pourian, et al., Prediction of crack initiation sites in alpha Ti-alloys microstructures under dwell-fatigue using Cellular Automaton simulation method. International Journal of Fatigue, 2016. 85: p. 85-97.

47. A. Fatemi and D.F. Socie, A Critical Plane Approach to Multiaxial Fatigue Damage Including out - of - Phase Loading. Fatigue \& Fracture of Engineering Materials \& Structures, 1988. 11(3): p. 149-165. 
48. K.O. Findley and A. Saxena, Low cycle fatigue in Rene 88DT at 650 C: crack nucleation mechanisms and modeling. Metallurgical and Materials transactions A, 2006. 37(5): p. 1469-1475. 